\title{
Multivariate robust modelling and optimization of cutting forces of the helical milling process of the aluminum alloy Al 7075
}

\author{
Robson Bruno Dutra Pereira ${ }^{1,3}$, Rodrigo Reis Leite ${ }^{2 *}$, Aline Cunha Alvim², Anderson Paulo de Paiva ${ }^{2}$, Pedro Paulo \\ Balestrassi $^{2}$, João Roberto Ferreira ${ }^{2}$, J. Paulo Davim ${ }^{3}$ \\ Corresponding author: *rodrigo_r112@ @otmail.com, +55 32 99168-3427 \\ ${ }^{1}$ Department of Mechanical Engineering - Industrial Engineering, Federal University of São João del Rei, 170 Frei \\ Orlando Square, São João del Rei, MG 36880000, Brazil \\ ${ }^{2}$ Institute of Industrial Engineering and Management, Federal University of Itajubá, 1303 BPS Avenue, Itajubá, MG \\ 37500-903, Brazil \\ ${ }^{3}$ Department of Mechanical Engineering, University of Aveiro, Campus Santiago, 3810-193 Aveiro, Portugal
}

\begin{abstract}
Helical milling is an advanced hole-making process and different approaches considering controllable variables have been presented addressing modelling and optimization of machining forces in helical milling. None of them considers the importance of the noise variables and the fact that machining forces components are usually correlated. Exploring this issue, this paper presents a multivariate robust modelling and optimization of cutting forces of the helical milling of the aluminum alloy Al 7075. For the study, the tool overhang length was defined as noise variable since in cavities machining there are specific workpiece geometries that constrain this variable; the controllable variables were axial feed per tooth, tangential feed per tooth and cutting speed. The cutting forces in the workpiece coordinate system were measured and the components in the tool coordinate system, i.e., the axial and radial forces, were evaluated. Since these two outcomes are correlated, the weighted principal component analysis was performed together with the robust parameter design to allow the multivariate robust modelling of the mean and variance equations. The normal boundary intersection method was used to obtain a set of Pareto robust optimal solutions related to the mean and variance equations of the weighted principal component. The optimization of the weighted principal component through the normal boundary intersection method was performed and the results evaluated in the axial and radial cutting forces components. Confirmation runs were carried out and it was possible to conclude that the models presented good fit with experimental data and that the Pareto optimal point chosen for performing the confirmation runs is robust to the tool overhang length variation. Finally, the cutting force models were also presented for mean and variance in the workpiece coordinate system in the time domain, presenting low error regarding the experimental test, endorsing the results.
\end{abstract}

\section{Keywords}

Helical milling; cutting forces; robust parameter design; multivariate mean square error; weighted principal component; normal boundary intersection.

\section{Introduction}

Machining of holes is a complex task when manufacturing a part. The conventional drilling process presents challenges such as the work material breakouts at hole exit, chip removal, heat dissipation difficulties and the increasing of thrust forces, which happens due to the extrusion process of material near the tool center point where the cutting velocity is close to zero. Burr on both entry and exit side of the hole, poor dimensional, geometrical and microgeometrical accuracy are likewise significant due to friction between tool, workpiece and chip [1-3].

Helical milling has a field of application in aerospace materials, such as aluminum, titanium alloys and CFRP materials, and is nowadays widely applied in industry for machining holes with high precision [4-7]. This process presents several benefits regarding the conventional drilling. Because of the helical milling kinematics, cylindrical holes of different diameters can be machined with the same tool. According to Iyer et al. [1], material removal at and near the hole center occurs by cutting rather than by extrusion, resulting in lower thrust force. Low burr formation, little delamination in CFRP, good chip transportation, good cutting fluid conditions, high dimensional, geometrical and microgeometrical accuracy compared with conventional drilling can be achieved $[1,8]$ facilitating the assembly of mechanical structures. The intermittent cutting process in helical milling is useful for cooling, reducing the risk of tool wear, breakage or failure arising from elevated temperature. Minimum quantity lubrication (MQL) and air blow could be employed to assist chip transport while in many drilling applications cutting fluid is used merely to flush the chips from the cutting zone [9].

\subsection{Cutting forces in helical milling}

The helical milling process is based on a rotating mill, which performs a helical trajectory. According to Fang et al. [10] and Tanaka et al. [11], in helical milling, besides the tool rotation around its own axis, with a related cutting velocity ( $v_{c}$ ) in $[\mathrm{m} / \mathrm{min}]$, the result of the synchronism between the axial feed of $z \mathrm{CNC}$ axis and the interpolation of the $x y$ axes 
generates the helical path. The borehole diameter $\left(D_{b}\right)$ is determined by the tool diameter $\left(D_{t}\right)$ and the helical diameter $\left(D_{h}\right)$, as shown in Fig. 1. This kinematics allows the generation of different borehole diameters with a single mill by varying the helical path diameter. The helical milling kinematics was previously described [12,13] and may be defined regarding tangential and frontal cut.

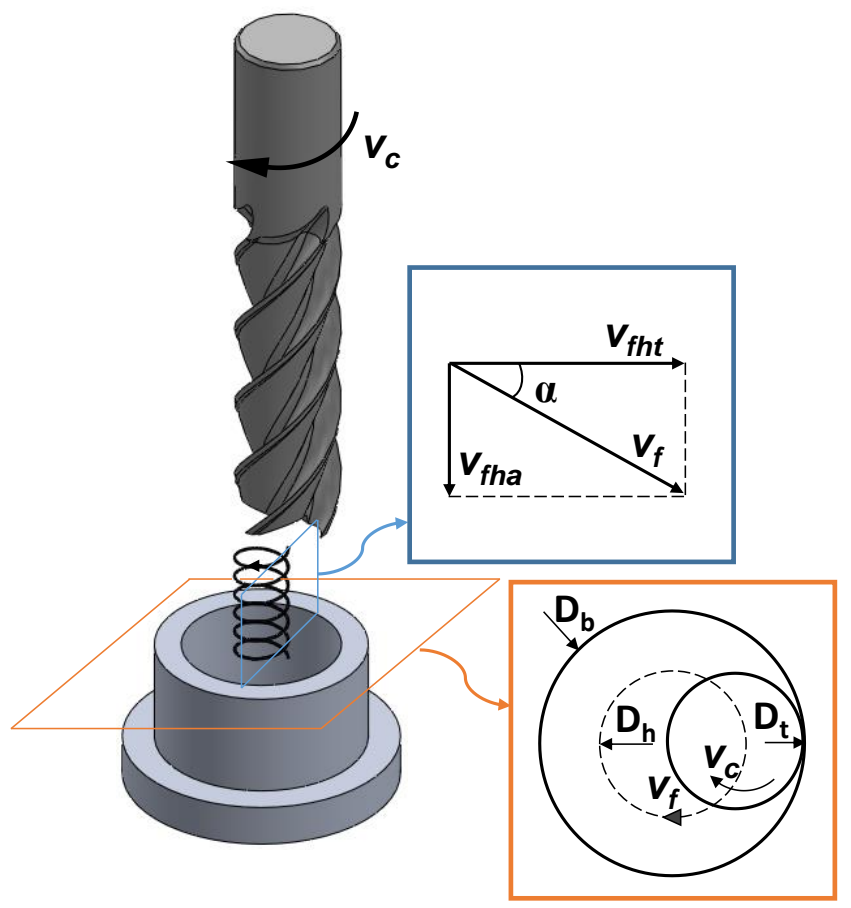

Fig. 1 Helical milling kinematics

As shown in Eq. (1), the helical feed velocity $\left(v_{f}\right)$ in $[\mathrm{mm} / \mathrm{min}]$ can be decomposed into the tangential feed velocity of the helix $\left(v_{f h t}\right)$ and the axial feed velocity of the helix $\left(v_{f h a}\right)$, both in $[\mathrm{mm} / \mathrm{min}]$. The axial feed velocity of the helix can be described considering the axial feed per tooth $\left(f_{z a}\right)$ in $[\mathrm{mm} /$ tooth], the number of teeth $(z)$ and the spindle rotation speed $(n)$ in [RPM], as in Eq. (2). In the circular trajectory, the tangential feed velocity of the helix $\left(v_{f h t}\right)-$ which is according to Pereira et al. [13] the circular velocity of the tool center point related to the helical diameter $\left(D_{h}\right)$ in $[\mathrm{mm}]-$ can be mathematically described in terms of the tangential feed velocity of the cutting edge $\left(v_{f t}\right)$ in $[\mathrm{mm} / \mathrm{min}]$, related to bore hole diameter $\left(D_{b}\right)$ in [mm], as in Eq. (3).The tangential velocity $\left(v_{f t}\right)$, expressed in Eq. (4), may be described in function of the tangential feed per tooth $\left(f_{z t}\right)$ in $[\mathrm{mm} /$ tooth], the number of teeth $(z)$ and the spindle rotation speed $(n)$ in [RPM].

$v_{f}=\sqrt{\left(v_{f h a}^{2}+v_{f h t}^{2}\right)}$

$v_{f h a}=f_{z a} \cdot z \cdot n$

$v_{f h t}=v_{f t} \cdot \frac{D_{h}}{D_{b}}$

$v_{f t}=f_{z t} \cdot z \cdot n$

In helical milling process, the helical pitch, Eq. (5), is the axial cutting depth $\left(a_{p}{ }^{*}\right)$ in [mm], which depends mathematically on the axial and tangential feed velocities. Then, it can be described in terms of the angle of the helix $(\alpha)$, expressed in Eq. (6). The axial cutting depth can be also described considering the axial and tangential feed per tooth, $f_{z a}$ and $f_{z t}$. This decomposition is important to address the effects of the feed on these directions on the cutting force components. 
$a_{p}^{*}=\tan (\alpha) \cdot \pi \cdot D_{h}=\frac{f_{z a} \cdot \pi \cdot D_{b}}{f_{z t}}$

$$
\alpha=\arctan \left(\frac{v_{f h a}}{v_{f h t}}\right)
$$

In cutting force prediction, it is relevant to define two coordinate systems to describe the process tool motion: the workpiece coordinate system and the tool coordinate system $[8,12]$. In the workpiece coordinate system, the coordinate axis directions $\mathrm{x}, \mathrm{y}$ and $\mathrm{z}$ are fixed whereas the tool coordinate system varies with tool rotation and orbital revolution. To address cutting forces in helical milling, it is important to take into consideration these two coordinate systems. The workpiece coordinate system is used for monitoring. The components in the tool coordinate system are important to understanding i) the influence of the cutting parameters in cutting forces and ii) the correlation among cutting forces and quality parameters, for instance, the dimensional deviation due to radial force.

Predicting cutting forces is important to assure energy economy, workpiece quality, tool integrity and to reduce machining damage $[14,15]$. Cutting forces has a relationship with power consumption and tool wear [16]. In the precision manufacturing of a part, minimizing cutting force levels allows obtaining high-quality parts by minimizing vibrations, tool wear and tool deflection. Different studies are mentioned in this section to summarize current results about cutting forces trends and modelling in the helical milling process.

Among cutting forces components in the helical milling process, the axial force $\left(F_{a}\right)$ acts along the $z$-axis while the radial force $\left(F_{r}\right)$ acts toward the tool center [17]. It is relevant to address $F_{r}$ because it may lead to tool radial deflection causing vibrations. This is related to geometrical and dimensional deviations and degradation of the surface finish of the hole [18].

Concerning tool cutting edges, the peripheral cutting edge generates mainly $F_{r}$, and the front cutting edge generates mainly both $F_{r}$ and $F_{a}$ [17]. $F_{r}$ is also called feed normal force $\left(F_{f N}\right)$ [12]. It is a resultant force that can be calculated through the components $F_{x}$ and $F_{y}$, which are cutting forces in the $x$ and $y$ directions on the $x y$ plane of the workpiece coordinate system, $F_{r}=\sqrt{F_{x}^{2}+F_{y}^{2}}$ [19]. $F_{a}$, measured according to the tool coordinate system, is equal to $F_{z}$, measured in the workpiece coordinate system [19].

Brinksmeier et al. [8] studied helical milling kinematics and modelled the undeformed chip geometry, considering peripheral and frontal cuts. Denkena et al. [12] assured that in the helical milling process, continuous and discontinuous cuts are presented in peripheral and frontal cut, respectively. This cutting mechanics leads to differences in cutting forces and temperature, generating force levels 10 times lower than force levels in drilling process $[9,20]$.

Denkena et al. [12] studied cutting forces components considering the tool coordinate system (feed force $F_{f}$ and feed normal force $F_{f N}$ ) on CFRP-titanium layer compounds in the helical milling. It was found that the axial feed per tooth increases contributes to the progress of feed force and feed normal force due to an increased pitch of the helical tool path. Consequently, this increases the height of the undeformed chip. The tangential feed per tooth increase leads to decreasing feed and feed normal forces due to a reduction of the height of the undeformed chip.

Shan et al. [20] studied the big pitch influence on machining forces components in the helical milling of the aerospace Al-alloy 6061. It was shown that the pitch increase leads to a growth in the axial cutting force component $F_{z}$ since the axial feed increases with pitch increment.

Liu et al. [21] analytically modelled the cutting forces on side cutting edges and on end cutting edges along the helical feed path considering the tangential and axial feeds of the tool, the spindle rotation, and the interaction between tool and workpiece. However, the experimental results showed percentage error levels from $6.36 \%$ to $11.8 \%$ of the simulation results.

Ventura and Hassui [14] modelled cutting forces in the helical milling using a circular insert, taking into consideration the tool contact angle and the respective depth of cut. The simulation of $F_{z}$ and $F_{y}$ presented similar profiles when compared to the experimental results, despite the observation of some errors, which according to the authors occurred mainly due to the dynamics of the machine and the used approximations.

Haiyan and Xuda [17] developed a mechanistic cutting force model to improve the cutting forces prediction in the helical milling of CFRP. It considered the fiber cutting angle according to helical milling cutting principles. The established model with the cutting forces coefficients seemed to be efficient to calculate relative accurate cutting forces in the helical milling of carbon fiber-reinforced polymers.

Wang et al. [19] conducted a comparative study involving stacks of CFRP/Ti versus CFRP and Ti alloy single plate in the helical milling process. Among the obtained results, during the machining of stacks of CFRP/Ti, the cutting force suddenly increased in machining of titanium alloy. That increase is significant if compared to CFRP in the stack and in the single titanium alloy layer. A good justification is the abrasive tool wear during CFRP machining, leading to the increase of cutting force, especially the axial cutting force $F_{z}$ in titanium stack machining. 
Rey et al. [22] developed cutting force models for the helical milling of Ti-6Al-4V titanium alloy with consideration to tool geometry and cutting conditions. They considered the chip geometry regarding cutting conditions and tool geometry and defined a cutting force model based on the instantaneous chip thickness. The models for $F_{z}$ and $F_{r}$ achieved errors of $18.8 \%$ and $5.35 \%$, respectively.

Li et al. [23] developed analytical cutting force models for side and bottom flutes with cutter runout in helical milling. The results showed the models considering the cutter runout presented errors of $6.09 \%, 10.72 \%$, and $13.59 \%$ in the $x, y$, and $z$ directions, which are better results than previous models.

Considering the referred results, it is necessary to obtain better models for cutting force components in the helical milling process. Furthermore, the error levels of the proposed analytical models are usually related to the environmental or noise factors (variables), i.e., the non-controllable factors. Modelling approaches that consider only controllable factors do not take into consideration part of data variability, relative to noise factors, without explanation. This can generate significant error levels in predicting cutting forces. In addition, cutting force components usually present high correlation structures, which can influence negatively the estimation of regression model coefficients. Therefore, multivariate robust modelling and optimization techniques need to be applied due to the multivariate nature of cutting forces and the presence of noise factors in the helical milling process.

Given the aforementioned discussion, the aim of this paper is to optimize the correlated responses axial and radial cutting forces components of the helical milling of the aluminum alloy $\mathrm{Al}$ 7075. The independent variables considered were: axial and tangential feed per tooth, cutting velocity and tool overhang length. In this line of reasoning, this work presents a multivariate robust modelling and optimization method (MMSE $\mathrm{W}_{-\mathrm{NBI}}$ ), which is based on the weighted principal component (WPC) technique, robust parameter design (RPD), response surface methodology (RSM), mean square error (MSE) and normal boundary intersection (NBI) optimization methods.

To deal with the correlation structure between the axial and radial cutting forces, the weighted principal component technique was performed yielding principal component scores, which represent the data set of the original correlated responses axial and radial cutting forces. Given these scores, the next step could be carried out, which was the robust parameter design approach and response surface methodology. Performing these techniques, response surface models for the mean and variance equations of the weighted principal component and the axial and radial cutting forces were obtained. As the mean square error optimization technique is an agglomeration strategy of two objective functions into one, leading to the same problems of the weighted sum method, the normal boundary intersection optimization method was applied to achieve an even spread of Pareto optimal solutions of the mean and variance equations of the weighted principal component; then, through the optimization of the mean and variance of the weighted principal component, the mean and variance of the original responses were also optimized. Moreover, confirmation runs were carried out to confirm the process robustness regarding the noise factor, tool overhang length.

In relation to cutting forces modelling, besides presenting multivariate and univariate cutting force models for mean and variance in the tool coordinate system, the present paper presents cutting force models for mean and variance equations in the workpiece coordinate system in the time domain. These models are also robust regarding the tool overhang length variation.

To allow robust design and optimization, the tool overhang length $\left(l_{t o}\right)$ was considered as noise factor because in cavity machining there are specific workpiece geometries that constrain the tool overhang length [24]. Then, it is not a control factor, i.e., it does not depend on the experimenter choice. Fig. 2(a) illustrates a mold with hole-making tasks, while Fig. 2(b) shows that if $l_{\text {to }}$ is not enough, the tool holder may collide with the workpiece during the helical milling process. There are several papers dealing with $l_{t o}$ effect, modelling and optimization in machining processes [25-27]. Quantifying the effect of $l_{\text {to }}$ on process responses is important, but minimizing its effect on process performance is essential because sometimes the tool overhang length must be set according to the workpiece geometry. It is necessary to select optimal process factors levels to optimize the multiple set of responses, making the process robust with consideration to the tool overhang length variation. To achieve this aim, $l_{t o}$ was controlled at the experimental phase to assure the cutting force robustness regarding its variation in a practical situation. 

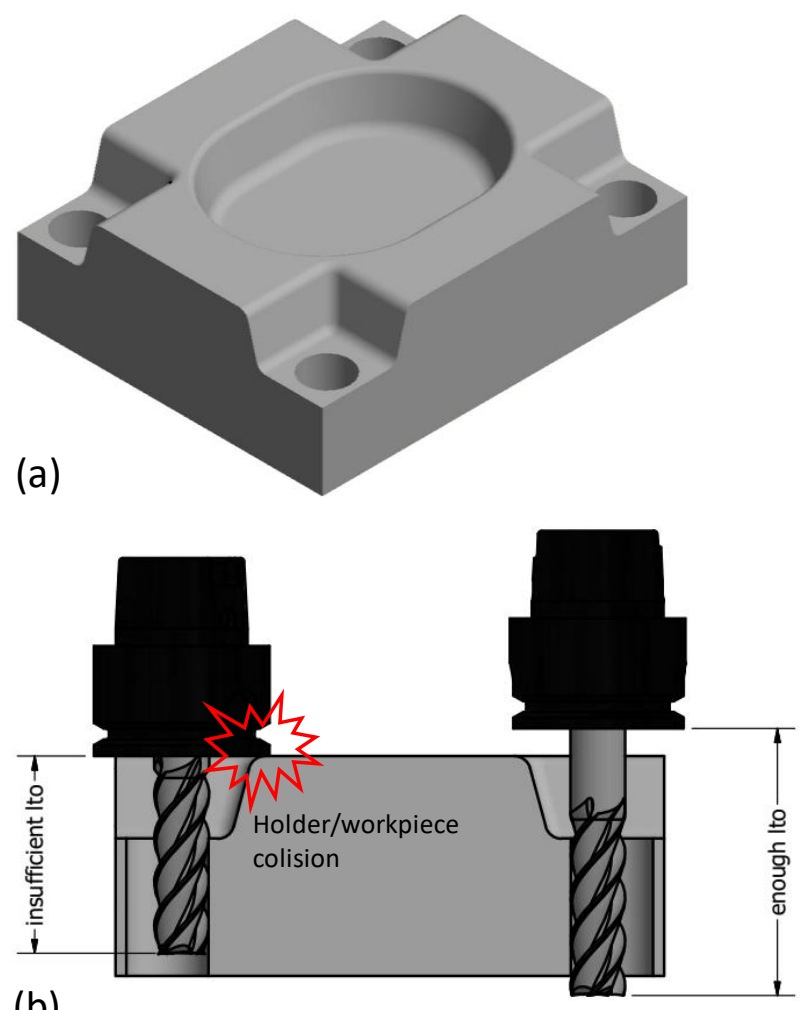

(b)

Fig. 2 (a) Mold with hole-making tasks and (b) Insufficient $\times$ enough tool overhang length $\left(l_{t o}\right)$ for the helical milling

The relevance of the present paper to industrial problems and situations is related to the fact that in on-line optimization situations, engineers deal with environmental factors and correlated process outcomes. In relation to process application, the helical milling brings a contribution in cutting forces modelling, considering the robustness regarding the tool overhang length variation, providing cutting force models in the tool and workpiece coordinate systems. Firstly, regarding noise factors, they are present in distinct manufacturing systems and are always referred as sources of errors that may not be easily controlled, leading to quality characteristics levels frequently outside upper and lower process control limits. Secondly, regarding correlated process outcomes, they may impact negatively the process of evaluating regression coefficients, leading to predicting models that do not correctly represent real process performances. Therefore, the MMSE $E_{W}-\mathrm{NBI}$ modelling and optimization method can easily take part of engineer's routine to deal with industrial problems related to optimization issues that contemplate environmental factors and correlated outcomes since the multivariate robust modelling and optimization method combines techniques capable of overcoming the aforementioned issues.

\section{Robust parameter design}

Robust parameter design (RPD) is a methodology to improve the quality of a product by minimizing the effect of causes of variation without eliminating them. This is achieved by optimizing process making its performance minimally sensitive to causes of variation [28]. RPD through Response Surface Methodology (RSM) was firstly proposed by Myers et al. [29] with the purpose of modelling the mean and variance equations separately. In the context of RPD, different RSM approaches are available and the two most popular are crossed arrays and combined arrays. Crossed array is the first RPD methodology and is made up of an inner array, considering controllable factors, and an outer orthogonal array containing noise factors. This methodology, however, does not consider the interactions between process and noise factors and requires a considerable number of runs [30].

Combined arrays were proposed by Welch et al. and Shoemaker et al. [31,32], and consists of running an experimental design containing both controllable and noise factors, eliminating the axial points related to noise factors as suggested by Myers et al. [29]. Taking into consideration controllable and noise factors into a single design leads to lower number of experimental runs and allows estimating the interaction between them [29,31-33].

Through a combined array based on a central composite design (CCD), a surface response model for the quality characteristics, in function of controllable and noise factors, $y(\mathbf{x}, \mathbf{z})$, can be obtained using ordinal or weighted least squares, OLS and WLS, respectively. Eq. (7) presents the full quadratic response surface model, where $\mathbf{x}$ and $\mathbf{z}$ are the vectors of controllable and noise factors, respectively. $1 \leq i \leq k$ and $1 \leq j \leq r$, where $k$ is the number of controllable factors and $r$ is the number of noise factors. $\beta, \lambda$ and $\delta$ are coefficients of the quadratic model. 


$$
y(\mathbf{x}, \mathbf{z})=\beta_{0}+\sum_{i=1}^{k} \beta_{i} x_{i}+\sum_{i=1}^{k} \beta_{i i} x_{i}^{2}+\sum_{i<j} \sum \beta_{i j} x_{i} x_{j}+\sum_{j=1}^{r} \lambda_{j} z_{j}+\sum_{i=1}^{k} \sum_{j=1}^{r} \delta_{i j} x_{i} z_{j}+\varepsilon
$$

Assuming independent noise factors with mean error equals to zero, variance $\sigma_{z_{j}}^{2}$, and that noise factors are uncorrelated with the random error $\varepsilon$, the mean and variance equations can be obtained in Eq. (8) and Eq. (9), respectively. $\sigma_{z_{j}}^{2}$ can be considered equal to 1 since the factor levels are scaled, and $\sigma^{2}$ is the residual error obtained in ANOVA. This approach is referred to as the propagation of error (POE). The variance equation is essentially a response surface with linear, quadratic, and second-order interaction terms.

$$
\begin{aligned}
& E_{z}[y(\mathbf{x}, \mathbf{z})]=f(\mathbf{x})=\beta_{0}+\sum_{i=1}^{k} \beta_{i} x_{i}+\sum_{i=1}^{k} \beta_{i i} x_{i}^{2}+\sum_{i<j} \sum \beta_{i j} x_{i} x_{j} \\
& V_{z}[y(\mathbf{x}, \mathbf{z})]=\sigma_{z_{j}}^{2}\left\{\sum_{j=1}^{r}\left[\frac{\partial y(\mathbf{x}, \mathbf{z})}{\partial z_{j}}\right]^{2}\right\}+\sigma^{2}
\end{aligned}
$$

\section{Mean square error optimization}

The Mean Square Error (MSE) optimization method was proposed by Box [34] and studied by Lin and Tu [35], and is expressed in Eq. (10), where $T$ is the utopia point obtained by $\operatorname{Min}_{\mathbf{x} \in \Omega}[\hat{y}(\mathbf{x})], \hat{y}(\mathbf{x})$ corresponds to the mean equation and $\hat{\sigma}^{2}$ is the variance equation. This approach, according to the authors, aims to minimize bias and variance and may be called dual response surface (DRS). Furthermore, mean and variance models can be obtained using crossed array or combined array approaches.

$$
\operatorname{Min}\left\{\operatorname{MSE}=(\hat{y}-T)^{2}+\hat{\sigma}^{2}\right\}
$$

Lin and Tu [35] also proposed the weighting of the mean and variance equations, as in Eq. (11), in order to allow the evaluation of various operational settings based on $w_{1}$ and $w_{2}$ values of interest.

$$
\operatorname{Min}\left\{M S E_{w}=w_{1}(\hat{y}-T)^{2}+w_{2} \hat{\sigma}^{2}\right\}
$$

In a scenario considering multiple modelled DRS, the optimization problem has multivariate nature and consists of a multivariate mean square error optimization. Thereby, Köksoy [36] extended the MSE criteria, proposing an agglutination strategy that optimizes the MSE of multiple responses, using a weighted sum or choosing the more important MSE function as objective function and setting the others as constraints. The agglutination strategy using weighted sum is exposed in Eq. (12).

$$
\operatorname{Min}\left\{M S E_{T}=\sum_{i=1}^{p} w_{i} M S E_{i}\right\}
$$

Since most of manufacturing processes presents multivariate nature, with significant correlation structure among responses, Paiva et al. [37] proposed a combination of MSE concepts with surface responses wrote in terms of principal component scores. This approach is the multivariate mean square error (MMSE), as illustrated in Eq. (13).

$\operatorname{Min}\left\{\operatorname{MMSE}_{i}=\left[\left(P C_{i}-\xi_{P C_{i}}\right)^{2}+\lambda_{i}\right]\right\}$

In Eq. (13), $P C_{i}$ is the regression model for the $i$-th principal component score, $\xi_{P C i}$ is the utopia point for this score, and $\lambda_{i}$ is its respective eigenvalue. For $p$ correlated responses, there are $p$ uncorrelated principal component scores; thereby the MMSE approach could be expanded considering the $k$ most representative scores of data variability, i.e., scores with $\lambda_{i} \geq 1$. Eq. (14) expresses this agglutination. 


$$
\begin{aligned}
\operatorname{Min} M M S E_{T} & =\left[\prod_{i=1}^{k}\left(M M S E_{i} \mid \lambda_{i} \geq 1\right)\right]^{\left(\frac{1}{k}\right)}=\left\{\prod_{i=1}^{k}\left[\left(P C_{i}-\zeta_{P C_{i}}\right)^{2}+\lambda_{i} \mid \lambda_{i} \geq 1\right]\right\}^{\left(\frac{1}{k}\right)} \\
i & =1,2, \ldots, k ; \quad k \leq p
\end{aligned}
$$

Paiva et al. [38] applied $\mathrm{MMSE}_{\mathrm{T}}$ on RPD considering a crossed array. Subsequently, Paiva et al. [39] extended the referred multivariate methodology for RPD considering a combined array. Through $p$ correlated response models, considering process and noise factors, response models for the most representative principal components scores were achieved. Appling the POE approach, mean and variance equations for principal components were obtained and this multivariate optimization approach is exposed in Eq. (15).

$$
\begin{aligned}
& \operatorname{Min} \operatorname{MMSE}(F i)(\mathbf{x})=\left\{\prod_{i=1}^{k}\left[\omega\left(E_{z}\left[P_{c}(\mathbf{x}, \mathbf{z})_{i}\right]-\xi_{P C z_{i}}\right)^{2}+(1-\omega)\left[\sigma_{z}^{2} \sum_{j=1}^{r}\left(\frac{\partial P_{c}(\mathbf{x}, \mathbf{z})_{i}}{\partial z_{j}}\right)+\sigma^{2}\right]\right]\right\}^{\left(\frac{1}{k}\right)} \\
& i=1,2, \ldots, k ; \quad k \leq p
\end{aligned}
$$

\section{Weighted principal components analysis (WPCA)}

In analyzing multi-response data, serious mistakes can be made. Fitting multi-response models without taking into consideration dependencies among responses can lead to difficulties, and the validity of the regression models under study may be denied [40]. Principal Component Analysis (PCA) may be applied to describe correlated responses in terms of uncorrelated principal components and to reduce problem dimension [41]. In the current context, an alternative approach for the multivariate mean square error optimization may consider all the $p$ principal component scores agglomerated into a single score weighted by their respective eigenvalues. The weighted principal component (WPC) resumes all $p$ components into one component without any loss of information [42], simplifying the modelling and optimization.

The main idea behind the principal component analysis is to reduce the $p$ original dimension in a system of $k \leq p$ coordinates considering a significant part of the correlated responses information. Considering $p$ correlated responses $\mathbf{y}^{T}=\left[y_{1}, y_{2}, \ldots, y_{p}\right]$, with mean vector $\boldsymbol{\mu}^{T}=\left[\mu_{1}, \mu_{2}, \ldots, \mu_{p}\right]$ and variance-covariance matrix $\boldsymbol{\Sigma}_{p x p}, p$ uncorrelated principal components can be achieved with the maximization of the variance of the linear combination $P C_{i}=\mathbf{e}_{i}^{T} \mathbf{y}=e_{1 i} y_{1}+e_{2 i} y_{2}+\ldots+e_{p i} y_{p}$, with $i=1,2, \ldots, p$, where $\mathbf{e}_{i}^{T}$ represents the standardized eigenvectors obtained from the eigenvalues, $\lambda_{1} \geq \lambda_{2} \geq \ldots \geq 0$, and from the variance-covariance matrix $\boldsymbol{\Sigma}_{p x p}$ [43]. These linear combinations describe the selection of a new coordinate system obtained by rotating the original system [44].

In a real situation, it is not possible to know the population parameters, and the responses may present different scales and units. Considering the estimated mean vector $\overline{\mathbf{y}}^{T}=\left[\bar{y}_{1}, \bar{y}_{2}, \ldots, \bar{y}_{p}\right]$ and the estimated correlation matrix $\mathbf{R}$, the $i$-th principal component estimation is defined as $\hat{P} C_{i}=[\mathbf{Z}]^{T}[\mathbf{E}]$, where $\mathbf{Z}$ is the standardized original responses matrix and $\mathbf{E}$ is the eigenvectors matrix. In this scenario, the exposed principles can be used to obtain the principal components estimates.

The weighted principal component score WPC is the linear combination of scores of the $p$ principal components, which considers its respective eigenvalues as scalars [42], as exposed in Eq. (16), in which the subscript " $s$ " means the experimental run number.

$$
W P C_{s}=\hat{\lambda}_{1} \hat{P} C_{1 s}+\hat{\lambda}_{2} \hat{P} C_{2 s}+\ldots+\hat{\lambda}_{r} \hat{P} C_{r s}
$$

\section{$5 \quad$ Normal boundary intersection method}

To overcome the drawbacks of the weighted sum method, Das and Dennis [45] proposed an approach called normal boundary intersection (NBI), which is a geometrically inspired scalarization method for solving non-linear multiobjective optimization problems, and its aim is to obtain evenly distributed Pareto optimal points, generating continuous frontiers regardless the weights attributed to the objective functions, which are process responses. In contrast to the weighted sum method, the NBI method finds a near-uniform spread of Pareto optimal solution options in the frontier [46]. In accordance with Zhang et al. [47], a set of points can be only considered as Pareto optimal if any improvement in 
one of the process responses lead to a setback in at least one of the other responses. Thus, the NBI method allows the experimenter a better exploitation of process parameters, facilitating analyses of the trade-off between a set of process responses.

The first step of the NBI method is to establish the payoff matrix $\boldsymbol{\Phi}[48,49]$. The payoff matrix is composed by the individual optimization of each objective function, evaluated in all objective functions involved in the multi-objective optimization problem. The payoff matrix can be written as in Eq. (17).

$$
\mathbf{\Phi}=\left[\begin{array}{ccccc}
f_{1}^{*}\left(\mathbf{x}_{1}^{*}\right) & \Lambda & f_{1}\left(\mathbf{x}_{i}^{*}\right) & \Lambda & f_{1}\left(\mathbf{x}_{m}^{*}\right) \\
\mathrm{M} & \mathrm{O} & & & \mathrm{M} \\
f_{i}\left(\mathbf{x}_{1}^{*}\right) & \Lambda & f_{i}^{*}\left(\mathbf{x}_{i}^{*}\right) & \Lambda & f_{i}\left(\mathbf{x}_{m}^{*}\right) \\
\mathrm{M} & & & \mathrm{O} & \mathrm{M} \\
f_{m}\left(\mathbf{x}_{1}^{*}\right) & \Lambda & f_{m}\left(\mathbf{x}_{i}^{*}\right) & \Lambda & f_{m}^{*}\left(\mathbf{x}_{m}^{*}\right)
\end{array}\right]
$$

In $\boldsymbol{\Phi}, \mathbf{x}_{i}^{*}$ represents a solution vector composed by values of process parameters that minimizes the $i$-th objective function $f_{i}$ individually. Thus, $f_{i}^{*}\left(\mathbf{x}_{i}^{*}\right)$ is the optimal solution for the $i$-th objective function, $i=1,2, \ldots, m$. The other solutions $f_{i}\left(\mathbf{x}_{i}^{*}\right)$, which are not on the main diagonal of $\boldsymbol{\Phi}$, are obtained by replacing $\mathbf{x}_{i}^{*}$ in the remaining functions. The set of individual optimal solutions that represents the main diagonal forms a vector called utopia point, $f^{U}=\left[f_{1}^{*}\left(\mathbf{x}_{1}^{*}\right), \ldots, f_{i}^{*}\left(\mathbf{x}_{i}^{*}\right), \ldots, f_{m}^{*}\left(\mathbf{x}_{m}^{*}\right)\right]^{T}$. On the other hand, the set of solutions that are most distant from the individual optimal of each objective function, i.e., $\operatorname{Max}\left\{f_{i}^{*}\left(\mathbf{x}_{i}^{*}\right), \ldots, f_{i}\left(\mathbf{x}_{m}^{*}\right)\right\}$, forms a vector known as pseudo nadir point, $f^{P N}=\left[f_{1}^{P N}, \ldots, f_{i}^{P N}, \ldots, f_{m}^{P N}\right]^{T}$. The normalization of each objective function should be made due to the nature of each process response. In doing so, the objective functions become dimensionless. Normalization can be performed as in Eq. (18).

$$
\bar{f}_{i}(\mathbf{x})=\frac{f_{i}(\mathbf{x})-f_{i}^{*}}{f_{i}^{P N}-f_{i}^{*}}
$$

The normalized payoff matrix $\overline{\mathbf{\Phi}}$ is in Eq. (19).

$$
\overline{\mathbf{\Phi}}=\left[\begin{array}{ccccc}
\bar{f}_{1}\left(\mathbf{x}_{1}^{*}\right) & \Lambda & \bar{f}_{1}\left(\mathbf{x}_{i}^{*}\right) & \Lambda & \bar{f}_{1}\left(\mathbf{x}_{m}^{*}\right) \\
\mathrm{M} & \mathrm{O} & & & \mathrm{M} \\
\bar{f}_{i}\left(\mathbf{x}_{1}^{*}\right) & \Lambda & \bar{f}_{i}\left(\mathbf{x}_{i}^{*}\right) & \Lambda & \bar{f}_{i}\left(\mathbf{x}_{m}^{*}\right) \\
\mathrm{M} & & & \mathrm{O} & \mathrm{M} \\
\bar{f}_{m}\left(\mathbf{x}_{1}^{*}\right) & \Lambda & \bar{f}_{m}\left(\mathbf{x}_{i}^{*}\right) & \Lambda & \bar{f}_{m}\left(\mathbf{x}_{m}^{*}\right)
\end{array}\right]
$$

According to Das and Dennis [45], the convex combinations formed by the sets of points of each row of $\overline{\boldsymbol{\Phi}}$ generate the convex hull of individual minima (CHIM), or Utopia line in bi-objective problems or Utopia plan in situations with more than two objective functions [46]. The Utopia line/plan is delimited by anchor points. An anchor point is the result of the individual optimization of only one objective function [46].

An optimization problem based on the NBI method can be written as in Eq. (20) [45].

$\operatorname{Max}_{(\mathbf{x}, t)} \quad t$

Subject to: $\overline{\mathbf{\Phi}} \boldsymbol{\beta}+t \hat{\mathbf{n}}=\overline{\mathbf{F}}(\mathbf{x})$

$$
\mathbf{x} \in \Omega
$$

$$
\begin{aligned}
& g_{j}(\mathbf{x}) \leq 0 \\
& h_{j}(\mathbf{x})=0
\end{aligned}
$$

Where $t \in R$ represents the distance between a point on CHIM and a point on the Pareto frontier and $\hat{\mathbf{n}}$ represents a unit vector normal to CHIM towards the origin that crosses a point $\overline{\mathbf{\Phi}} \boldsymbol{\beta}_{i}$. The weight $\boldsymbol{\beta}$ is a vector containing the 
degree of importance that is intended to be linked to each objective function, $\boldsymbol{\beta}=\left[\beta_{1}, \ldots, \beta_{i}, \ldots, \beta_{m}\right]^{T}, 0 \leq \beta_{i} \leq 1$, $\sum_{i=1}^{m} \beta_{i}=1, i=1,2, \ldots, m . \overline{\mathbf{F}}(\mathbf{x})$ is a vector containing the normalized objective functions,

$\overline{\mathbf{F}}(\mathbf{x})=\left[\bar{f}_{1}\left(\mathbf{x}^{*}\right), \ldots, \bar{f}_{i}\left(\mathbf{x}^{*}\right), \ldots, \bar{f}_{m}\left(\mathbf{x}^{*}\right)\right]^{T}$. Thus, $\overline{\boldsymbol{\Phi}} \boldsymbol{\beta}+t \hat{\mathbf{n}}$ represents a set of points normal to CHIM. According to Das and Dennis [50], the point of intersection between the normal $\overline{\mathbf{\Phi}} \boldsymbol{\beta}+t \hat{\mathbf{n}}$ and the boundary of the feasible region (Pareto frontier) closest to the origin is the global optimum. This global optimum will correspond to the maximization of the distance between a point on CHIM and the Pareto frontier.

\section{Multivariate RPD through WPCA and NBI}

It is possible to obtain a response model considering the weighted principal component scores $W P C_{s}$ by defining an experimental procedure based on a central composite design, which combines the process and noise factors. The response model is shown in Eq. (21).

$W P C(\mathbf{x}, \mathbf{z})=\beta_{0}+\sum_{i=1}^{k} \beta_{i} x_{i}+\sum_{i=1}^{k} \beta_{i i} x_{i}^{2}+\sum_{i<j} \sum \beta_{i j} x_{i} x_{j}+\sum_{j=1}^{k} \lambda_{j} z_{j}+\sum_{i=1}^{k} \sum_{j=1}^{r} \delta_{i j} x_{i} z_{j}+\varepsilon$

Based on POE principles, mean and variance equations can be obtained for WPC, through Eq. (22) and Eq. (23). These equations represent, respectively, the multivariate mean and variance of the $p$ correlated responses.

$E_{z}[W P C(\mathbf{x}, \mathbf{z})]=E_{z}(\mathbf{x})$

$V_{z}[W P C(\mathbf{x}, \mathbf{z})]=V_{z}(\mathbf{x})=\sigma_{z_{j}}^{2}\left\{\sum_{j=1}^{r}\left[\frac{\partial W P C(\mathbf{x}, \mathbf{z})}{\partial z_{j}}\right]^{2}\right\}+\sigma^{2}$

By using Eq. (22) and Eq. (23), a multivariate mean square error criterion based on the weighted principal component scores may be presented, as in Eq. (24). The constraint $\mathbf{x}^{\mathbf{T}} \mathbf{x} \leq \rho^{2}$ is related to the experimental region, $\xi_{W P C z}$ is the utopia point of $E_{z}[W P C(\mathbf{x}, \mathbf{z})]$ obtained by $\operatorname{Min}_{\mathbf{x} \in \Omega}\left\{E_{z}[W P C(\mathbf{x}, \mathbf{z})]\right\}$. Through this approach, the multivariate bias and variance can be minimized.

$\operatorname{Min}_{M} \operatorname{MSE}_{W}=\left\{\omega\left(E_{z}[W P C(\mathbf{x}, \mathbf{z})]-\xi_{W P C z}\right)^{2}+(1-\omega)\left[\sigma_{z_{j}}^{2} \sum_{j=1}^{r}\left(\frac{\partial W P C(\mathbf{x}, \mathbf{z})}{\partial z_{j}}\right)^{2}+\sigma^{2}\right]\right\}$

subject to: $\mathbf{x}^{\mathbf{T}} \mathbf{x} \leq \rho^{2}$

Paiva et al. [51] applied similar criteria in modelling a response surface for WPC considering only controllable factors. This approach presents a robust optimization MMSE $\mathrm{w}_{\mathrm{w}}$ considering WPC modelling based on combined arrays and on the POE approach to derive mean and variance equations. The MMSE $E_{W}$ proposed approach in Eq. (24) can be used to generate trade-off solutions for the mean and variance dual optimization problem. However, this approach is a weighted sum of mean and variance as well as the approaches in Eqs.10-15.

Due to the shortcomings of the weighted sum method in generating an even spread of Pareto optimal points, the NBI method may be applied together with the MMSEw method, depicted in Eq. (24). Considering the bi-objective NBI problem applied to the trade-off problem between Eq. (22) and Eq. (23), a payoff matrix $\boldsymbol{\Phi}$ is initially constructed based on optimal points of each isolated objective function. The solutions that minimize $E_{z}(\mathbf{x})$ and $V_{z}(\mathbf{x})$ are, respectively, $\mathbf{x}_{E}^{*}$ and $x_{V}^{*}$. The pay-off matrix for this specific dual problem is in Eq. (25).

$\boldsymbol{\Phi}=\left[\begin{array}{cc}E_{z}^{*}\left(\mathbf{x}_{E}^{*}\right) & E_{z}^{P N}\left(\mathbf{x}_{V}^{*}\right) \\ V_{z}^{P N}\left(\mathbf{x}_{E}^{*}\right) & V_{z}^{*}\left(\mathbf{x}_{V}^{*}\right)\end{array}\right]$ 
The elements of the payoff matrix will compose the anchor points, i.e., the extreme values of the Pareto frontier. $E_{z}^{*}\left(\mathbf{x}_{E}^{*}\right)$ and $V_{z}^{*}\left(\mathbf{x}_{V}^{*}\right)$ are utopia points, i.e., optimal values obtained through the optimization of $E_{z}(\mathbf{x})$ and $V_{z}(\mathbf{x})$, respectively. The worst results for these functions are called pseudo nadir points and are labeled $E_{z}^{P N}\left(\mathbf{x}_{V}^{*}\right)$ and $V_{z}^{P N}\left(\mathbf{x}_{E}^{*}\right)$. These extreme values on the solution space can be used to normalize the objective functions, as expressed in Eq. (26) and Eq. (27).

$$
\begin{aligned}
& \bar{E}_{z}(\mathbf{x})=\frac{E_{z}(\mathbf{x})-E_{z}^{*}\left(\mathbf{x}_{E}^{*}\right)}{E_{z}^{P N}\left(\mathbf{x}_{V}^{*}\right)-E_{z}^{*}\left(\mathbf{x}_{E}^{*}\right)} \\
& \bar{V}_{z}(\mathbf{x})=\frac{V_{z}(\mathbf{x})-V_{z}^{*}\left(\mathbf{x}_{V}^{*}\right)}{V_{z}^{P N}\left(\mathbf{x}_{E}^{*}\right)-V_{z}^{*}\left(\mathbf{x}_{V}^{*}\right)}
\end{aligned}
$$

Finally, the solution for this two-objective NBI optimization problem, called MMSE ${ }_{W}-\mathrm{NBI}$, can be exposed as in Eq. (28).

$$
\begin{aligned}
& \operatorname{Min} \bar{E}_{z}(\mathbf{x}) \\
& \text { s.t. }: \bar{E}_{z}(\mathbf{x})-\bar{V}_{z}(\mathbf{x})+2 w_{i}-1=0 \\
& g_{j}(\mathbf{x}) \geq 0 \\
& 0 \leq \omega_{i} \leq 1
\end{aligned}
$$

The MMSE $E_{W}$ NBI problem needs to be solved iteratively considering different weights. The space between two consecutive weights is defined as a constant $\delta$ [52]. For a bi-objective case, the number of Pareto optimal solutions ( $n$ ) may be calculated as $n=\delta^{-1}+1$. Thus, it is possible to obtain weights for the optimization problem presented in Eq. (29) as $w_{i}=(i-1) \delta, i=1,2, \ldots, n$. Therefore, $w_{1}=0, w_{2}=\delta, w_{2}=\delta, \ldots, w_{n}=1$.

Explicitly, the MMSE $\mathrm{W}_{\mathrm{W}} \mathrm{NBI}$ method, proposed in this paper, in terms of mean and variance of the weighted principal component, Eq. (29), allows obtaining equispaced Pareto frontiers, considering the mean and variance equations of the WPC response model, which represents a set of correlated responses without loss of information. In Eq. (29), the symbol $\psi$ indicates that the value of the variance equation between brackets is in the normalized form.

$$
\begin{aligned}
& \operatorname{Min} \bar{E}_{z}[\operatorname{WPC}(\mathbf{x}, \mathbf{z})] \\
& \text { s.t. : } \bar{E}_{z}[W P C(\mathbf{x}, \mathbf{z})]-\left[\sigma_{z_{j}}^{2} \sum_{j=1}^{r}\left(\frac{\partial W P C(\mathbf{x}, \mathbf{z})}{\partial z_{j}}\right)^{2}+\sigma^{2}\right]^{\psi}+2 w_{i}-1=0 \\
& \mathbf{x}^{\mathbf{T}} \mathbf{x} \leq \rho^{2} \\
& 0 \leq w_{i} \leq 1
\end{aligned}
$$

The MMSE ${ }_{W}-N B I$ method may be referred as a new procedure to optimize bias and variance of multivariate data without loss of information. The bias $\omega\left(E_{z}[W P C(\mathbf{x}, \mathbf{z})]-\xi_{W P C}\right)^{2}$ is not optimized explicitly in the objective function of the formulation in Eq. (29). Nevertheless, as the target $\xi_{W P C z}$ is obtained through $\operatorname{Min}_{\mathbf{x} \in \Omega}\left\{E_{z}[W P C(\mathbf{x}, \mathbf{z})]\right\}$, the proposed method in Eq. (29) will naturally minimize the bias and may be considered an alternative to the multivariate robust mean square error optimization to achieve an even spread of solutions for the trade-off between mean and variance of multivariate data without loss of information.

Lopes et al. [53] proposed a similar approach for robust modelling and optimization of the arithmetic average surface roughness and the maximum roughness height. However, the present paper proposes to the experimenter the possibility of using the weighted principal component technique, avoiding loss of information, i.e., $100 \%$ of the variancecovariance structure of the original set of process outcomes is taken into consideration to estimate regression coefficients that consider all the variance-covariance influence on the response surface models. Besides that, applying the $\mathrm{MMSE}_{\mathrm{W}}-$ NBI method on the helical milling is an important novelty since researches involving statistical influence of noise factors and correlated outcomes on this hole-making process has not been widely studied yet.

To solve the nonlinear programming problems (NLP) proposed in Eq. (24) and Eq. (29), several algorithms can be applied. One alternative algorithm is the generalized reduced gradient (GRG) $[38,54]$. 
To reach a robust approach to modelling and optimization of cutting forces in the helical milling process, the new proposed MMSE $\mathrm{W}_{\mathrm{W}} \mathrm{NBI}$ method is applied. The practical objective is to achieve cutting force models robust to the tool overhang length variation in the helical milling, i.e., in function of cutting parameters. Robustness is reached by achieving cutting parameter levels which are insensitive to the tool overhang length variation. This will allow a good fit of the cutting force models with experimental results in the tool and workpiece coordinate systems. The proposed approach is the first application of multivariate robust modelling and optimization in the helical milling process.

\section{$7 \quad$ Experimental procedure}

This work is a sequence of a previous work on modelling and optimization of the helical milling process of the aluminum alloy $\mathrm{Al} 7075$ [55]. In this referred first work, three outcomes were considered: axial cutting force component $\left(F_{z}\right)$, total roundness $\left(R o n_{t}\right)$ and material removal rate $(M R R)$. However, in this previous publication, only $F_{z}=F_{a}$ was presented with consideration to cutting forces components. The radial cutting force $F_{r}$, measured in the $x y$ plane, was not considered in that work since the intention was not only the modelling of cutting forces of the helical milling. In the present work, a statistically based approach is proposed for multivariate robust modelling and optimization of cutting forces of the helical milling process, without loss of information. When dealing with cutting forces modelling and optimization in the helical milling, it is important to consider $F_{a}$, which is related to the axial direction and is generally the main cutting force component in terms of magnitude, and $F_{r}$, which is measured in the circular plane, and consequently may be related to dimensional and geometrical deviations. Besides considering the cutting forces components in the tool coordinate system, i.e., $F_{a}$ and $F_{r}$, the present approach provides models for the cutting force components in the workpiece coordinate system $F_{x}, F_{y}$, and $F_{z}$. In these two viewpoints, robust models are provided, mean and variance models, considering the propagation of error regarding the tool overhang length.

$F_{a}$ and $F_{r}$ were modelled in function of three controllable variables $(\mathrm{CV})$ and one noise variable (NV) that can be seen in Table 1 along with their symbols, units, and levels. The tool overhang length was chosen as noise variable because it can be controlled only in an experimental scenario; on the other hand, it cannot be controlled at a production scenario since in molds and die machining there are workpieces geometries which constrain the tool overhang length, to avoid collisions between tool system and workpiece/fixture. Fig. 3 illustrates the setup of the three tool overhang length levels. Consequently, the RPD-RSM methodology is applied in this work to make $F_{a}$ and $F_{r}$ less sensitive to the tool overhang length variation. It is important to study the robustness of the tool overhang length in relation to cutting forces in helical milling, especially regarding $F_{r}$, since the tool overhang length is related to tool deflection, which may cause vibrations, and consequently dimensional, geometrical and microgeometrical errors [17]. Moreover, the variables presented in Table 1 were also taken into consideration to model $F_{r}$ and the mean and variance equations of the weighted principal component.
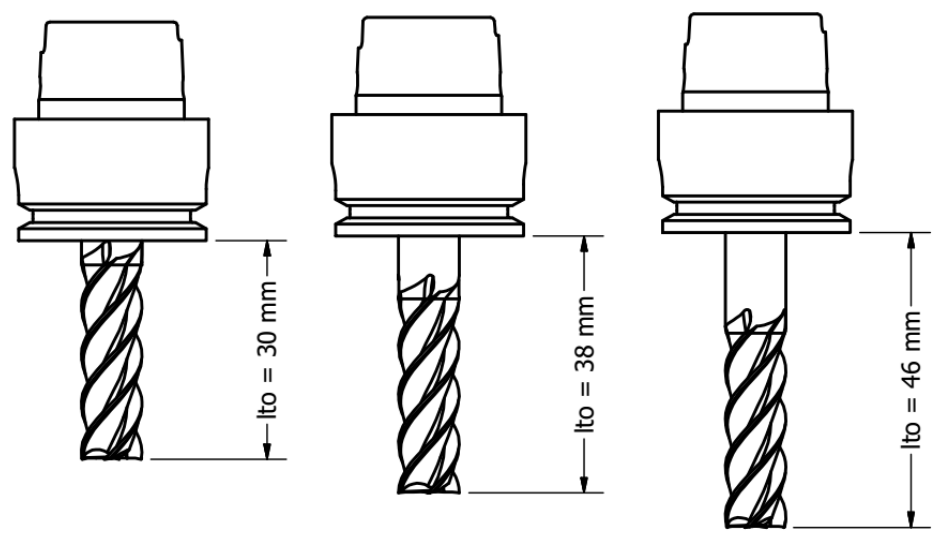

Fig. 3 Tool overhang length setup

Table 1 Controllable and noise variables. Adapted from [55] with permission from Elsevier, license number 4153600491710

\begin{tabular}{lllllllll}
\hline Type of variables & Variables & Symbols & Units & \multicolumn{2}{l}{ Levels } & & \\
\cline { 5 - 8 } & & & & -2 & -1 & 0 & 1 \\
\hline $\mathrm{CV}$ & Axial feed per tooth & $f_{z a}$ & $\mu \mathrm{m} /$ tooth & 0.5 & 4 & 7.5 & 11 & 14.5 \\
$\mathrm{CV}$ & Tangential feed per tooth & $f_{z t}$ & $\mu \mathrm{m} /$ tooth & 15 & 40 & 65 & 90 & 115 \\
$\mathrm{CV}$ & Cutting velocity & $v_{c}$ & $\mathrm{~m} / \mathrm{min}$ & 10 & 30 & 50 & 70 & 90 \\
$\mathrm{NV}$ & Tool overhang length & $l_{t o}$ & $\mathrm{~mm}$ & - & 30 & 38 & 46 & - \\
\hline
\end{tabular}


With the variables and levels presented in Table 1, a central composite design with 26 runs was used. Then, $n_{F}=$ $2^{k+r}=2^{3+1}=16$ factorial points (coded level $= \pm 1$ ) were carried out taking into consideration controllable and noise variables. The factorial points are important to study main effects of process and factors and process $\times$ process and process $\times$ noise interactions. The propagation of error considers not only the isolated effect of noise variable but also the effect of noise on controllable variables. $n_{A}=2 \times k=2 \times 3=6$ axial points (coded levels $= \pm 2$ ) were conducted considering only process variables to estimate their quadratic effects. Finally, $n_{C}=4$ center points (coded level $=0$ ) were conducted to allow an independent estimation of experimental error and support the calculation of quadratic terms.

The axial runs corresponding to the tool overhang length (noise variable) were not considered, and the experiments were conducted in random order to yield the most accurate analyses of data.

The axial distance for this design is $\rho=\left(2^{4}\right)^{1 / 4}=2$. However, when considering the process experimental space, since mean and variance equations are in function of only controllable parameters, the axial distance will become $\rho=$ $\left(2^{3}\right)^{1 / 4}=1.682$, which is the radius of the spherical constraint to the controllable variables in the optimization problem; thus, the solutions will be forced by the spherical constraint to fall within the experimental solution.

Here, besides $F_{a}, F_{r}$ was calculated considering the results of $F_{x}$ and $F_{y}$. Since $F_{a}$ and $F_{r}$ are statically correlated, the new non-linear multivariate robust modelling and optimization method (MMSE ${ }_{\mathrm{W}} \mathrm{NBI}$ ) can be performed to optimize both responses through the optimization of mean and variance equations of the weighted principal component, $E_{z}[W P C(\mathbf{x}, \mathbf{z})]$ and $V_{z}[W P C(\mathbf{x}, \mathbf{z})]$, respectively, without loss of information.

A CNC machining center was used in the experimental tests, with the following characteristics: Romi Discovery 560 , maximum power of $15 \mathrm{~kW}$, spindle speed of 10000 RPM, numerical control Siemens 810D CNC Sinumerik. The cutting tool used for machining the workpieces was an uncoated solid carbide end mill with the following characteristics: $10 \mathrm{~mm}$ diameter, 4 teeth, and $30^{\circ}$ helix angle. The cutting fluid was: soluble biodegradable emulsifiable oil for machining Bio 100 from Biolub Química ${ }^{\circledR}$ applied on wet condition.

The characteristics of the aluminum alloy Al 7075 workpieces were: $23 \mathrm{~mm}$ diameter, $14 \mathrm{~mm}$ height. Boreholes with $15 \mathrm{~mm}$ diameter were obtained in helical milling tests. Due to the ratio between mechanical resistance and density, the aluminum alloy Al 7075 has been widely applied in the aircraft industry due to its high mechanical strength to specific mass.

The machining components $F_{a}$ and $F_{r}$ were measured with a stationary piezoelectric dynamometer, model Kistler $^{\circledR} 9272$ with four components and signal amplifier. The three components $F_{x}, F_{y}$, and $F_{z}$ in the workpiece coordinate system were measured during the tests. Fig. 4 shows the experimental setup (a), helical milling test (b), nomenclature (c), and graphical results of cutting forces (d).
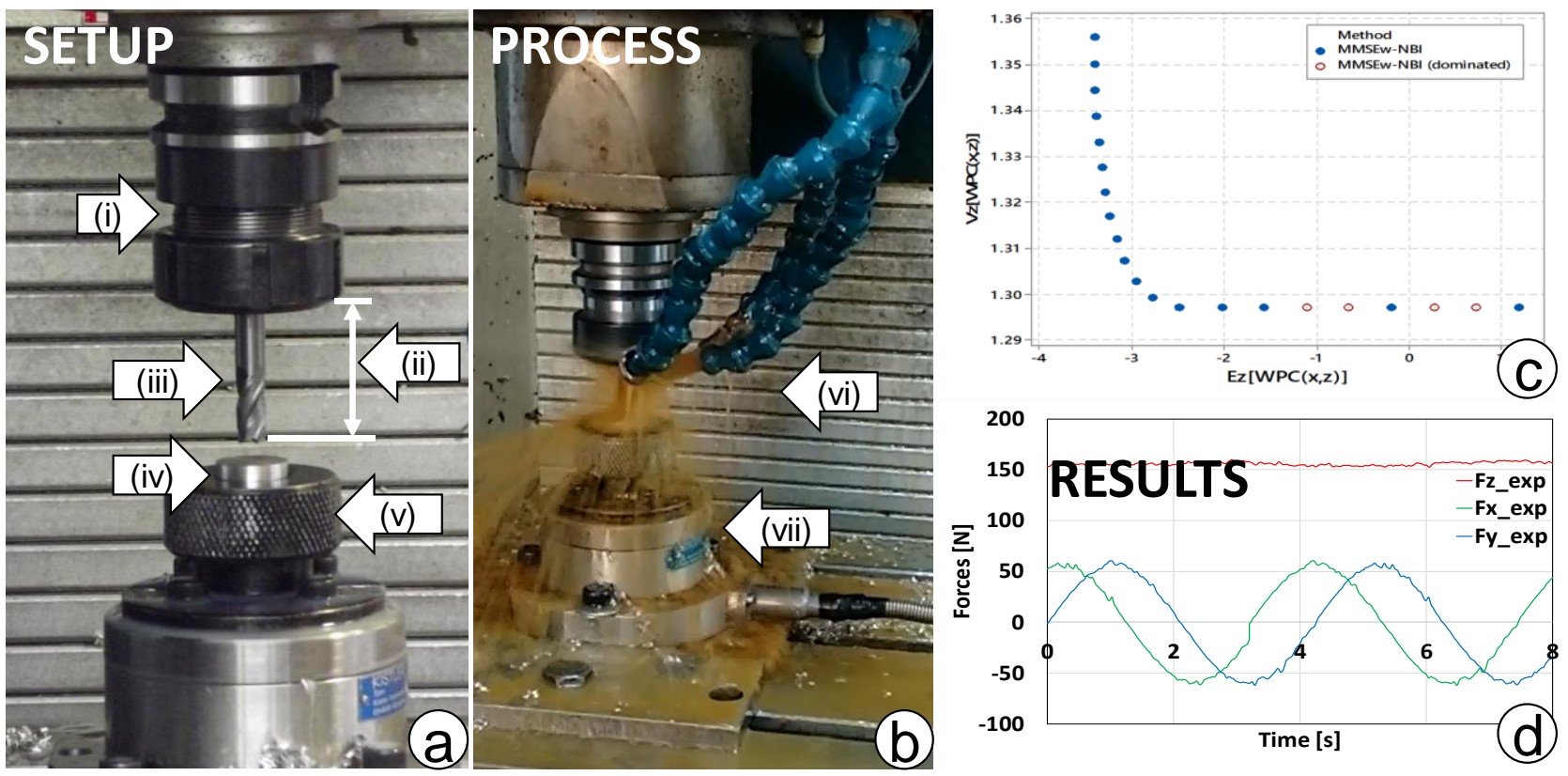

Fig. 4 Experimental procedure (i) Holder; (ii) Tool overhang length; (iii) Tool; (iv) Workpiece; (v) Fixture; (vi) Holemaking through helical milling; (vii) Dynamometer

In this paper, the following software were used for modelling and optimization: MS-Excel ${ }^{\circledR}$, Minitab $^{\circledR}$, and Matlab ${ }^{\circledR}$. The routine of the MMSE $\mathrm{W}_{\mathrm{W}} \mathrm{NBI}$ method is illustrated in Fig. 5. In Fig. 5, it is seen that if there is no correlation among outcomes, it should be performed univariate analysis and an optimization method should be chosen to solve the nonlinear optimization problem; this process is illustrated by the symbol $(+)$, indicating that this is another process. 
Considering Fig. 5, the procedure for the $\mathrm{MMSE}_{\mathrm{W}}-\mathrm{NBI}$ method can be described for replication and better understanding through the following steps:

Step 1: Define the experimental design

Define a central composite design combining control and noise factors. Run the experimental design, measure the responses and store the results.

Step 2: Evaluate the correlation among outcomes

Before starting PCA, it is indispensable to calculate the correlation among responses. It could be done by analyzing the Pearson correlation coefficient together with the $p$-value for each pair of responses.

Step 3: Run the principal component analysis

In the case of correlation, run the PCA analysis for the correlated responses using the correlation matrix R. Store the scores of the $p$ principal components and their respective eigenvalues and eigenvectors.

Step 4: Define the weighted principal component scores

Define the $W P C_{s}$ scores as linear combinations of the scores of the $p$ principal components weighted by their respective eigenvalues, as in Eq. (16). Store the $W P C_{s}$ scores.

Step 5: Build the WPC response model in function of controllable and noise factors

Use ordinal (OLS) or weighted least squares (WLS) methods to build a complete response model for WPC(x,z) through Eq. (21), considering linear terms, interaction between noise and control factors and quadratic terms for controllable factors.

\section{Step 6: Define mean and variance equations}

Through RPD, define mean and variance equations of the weighted principal component, $E_{z}[W P C(\mathbf{x}, \mathbf{z})]$ and $V_{z}[W P C(\mathbf{x}, \mathbf{z})]$, respectively, as defined in Eq. (22) and Eq. (23). Henceforth, $E_{z}[W P C(\mathbf{x}, \mathbf{z})]$ might be referenced as $E_{z}(\mathbf{x})$ and $V_{z}[W P C(\mathbf{x}, \mathbf{z})]$ might be referenced as $V_{z}(\mathbf{x})$.

Step 7: Define the payoff matrix

Obtain the utopia points $E_{z}^{*}\left[\mathbf{x}_{E_{z}(W P C)}^{*}\right]$ and $V_{z}^{*}\left[\mathbf{x}_{V_{z}(W P C)}^{*}\right]$ through the constrained optimization problems $\operatorname{Min}_{\mathbf{x}^{T} \mathbf{x} \leq \rho^{2}}\left\{E_{z}[W P C(\mathbf{x}, \mathbf{z})]\right\}$ and $\operatorname{Min}_{\mathbf{x}^{T} \mathbf{x} \leq \rho^{2}}\left\{V_{z}[W P C(\mathbf{x}, \mathbf{z})]\right\}$, respectively. The solution vector $\mathbf{x}_{E_{z}(W P C)}^{*}$ corresponds to the best individual values of $f_{z a}, f_{z t}$ and $v_{c}$ that minimizes $E_{z}(\mathbf{x})=E_{z}[W P C(\mathbf{x}, \mathbf{z})]$. In the same line of reasoning, $\mathbf{x}_{V_{z}(W P C)}^{*}$ contains the best individual values of the controllable variables that minimizes $V_{z}(\mathbf{x})=V_{z}[W P C(\mathbf{x}, \mathbf{z})]$. Using $\mathbf{x}_{E_{z}(W P C)}^{*}$ and $\mathbf{x}_{V_{z}(W P C)}^{*}$, define the pseudo nadir points $E_{z}^{P N}\left[\mathbf{x}_{V_{z}(W P C)}^{*}\right]$ and $V_{z}^{P N}\left[\mathbf{x}_{E_{z}(W P C)}^{*}\right]$. Build the payoff matrix as in Eq. (25).

\section{Step 8: Normalization}

Using the payoff matrix, normalize the mean and variance functions of the weighted principal component as well as the payoff matrix, as proposed in Eq. (26) and Eq. (27).

Step 9: Choose weights

Before carrying out the MMSE ${ }_{W}-\mathrm{NBI}$ method, the weights should be calculated. First, calculate the number of Pareto optimal solutions ( $n$ ) according to the desired increment $\delta$ between weights. Given $n$ and $\delta$, it is possible to obtain weights $w_{i}$, which are in Eq. (29), as $w_{i}=(i-1) \delta, i=1,2, \ldots, n$. For instance, in this paper, $w_{n}=1$ forces the optimization problem to achieve the best setting of $f_{z a}, f_{z t}$ and $v_{c}$ inherent to the best level of $E_{z}[W P C(\mathbf{x}, \mathbf{z})]$.

\section{Step 10: Run the MMSEw-NBI method}

Solve the MMSE ${ }_{W}-N B I$ method iteratively for different weights, as defined in Eq. (29), using the GRG algorithm. Build a Pareto frontier with equispaced Pareto solutions considering the Pareto optimal points for $E_{z}(\mathbf{x})=E_{z}[W P C(\mathbf{x}, \mathbf{z})]$ and $V_{z}(\mathbf{x})=V_{z}[W P C(\mathbf{x}, \mathbf{z})]$.

\section{Step 11: Confirmation runs}

Choose one or more Pareto optimal points according to preferences of the decision maker. Run tests considering a robust optimal vector, for instance $\mathbf{x}_{w_{i}}^{*}=\left(f_{z a}^{*}, f_{z t}^{*}, v_{c}^{*}\right)$, for different noise factors conditions. Test the process robustness for the Pareto optimal conditions and the proximity for the measured responses with the predicted values. 


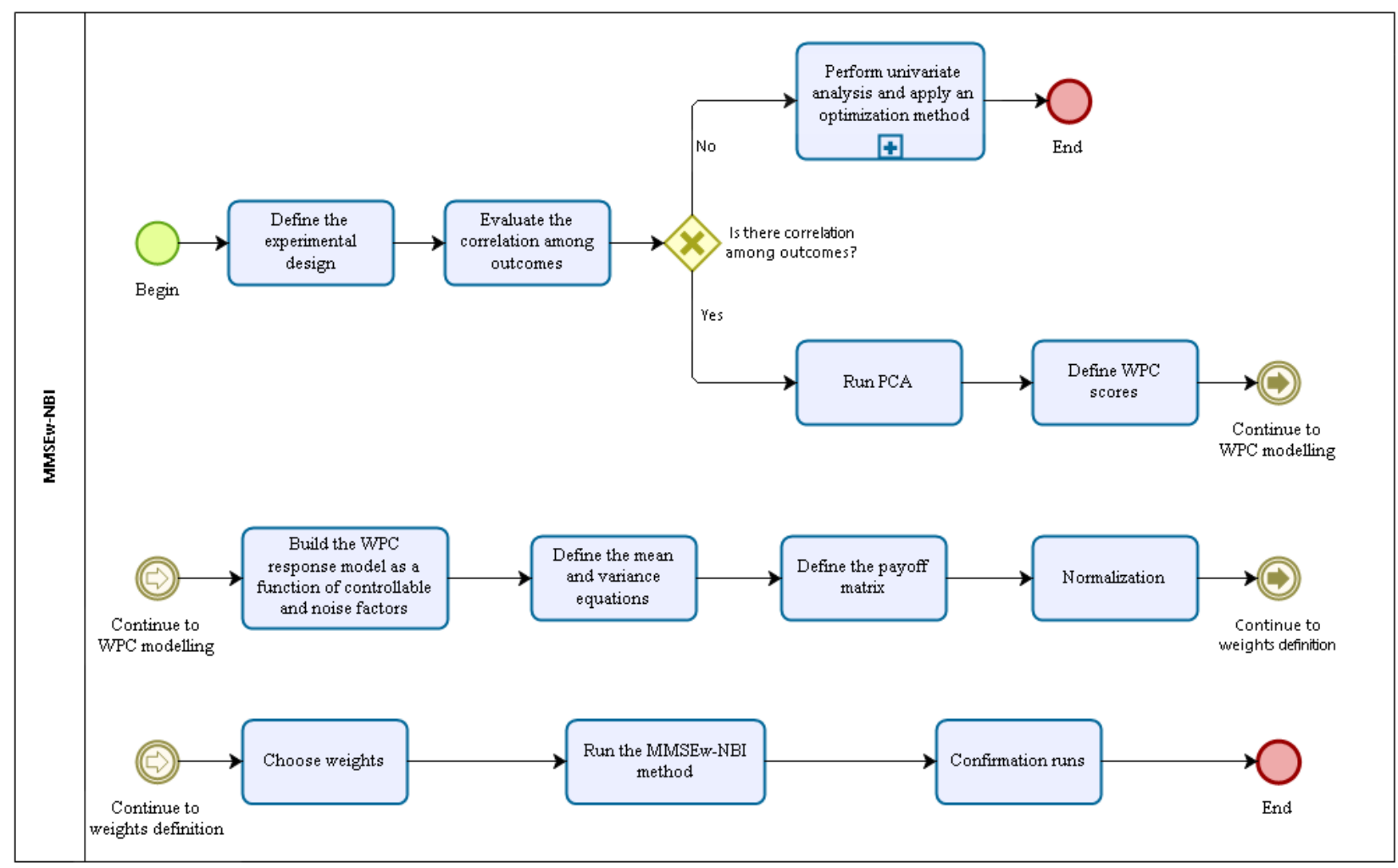

Fig. 5 Routine of the MMSEw-NBI method

\section{$8 \quad$ Results and discussion}

\subsection{Experimental design}

Table 2 presents the experimental design with cutting force results for the helical milling tests in the aluminum alloy $\mathrm{Al}$ 7075. Here, in terms of experimental results, the novelty is the response $F_{r}$ measured in the $x y$ plane, which may cause dimensional, geometrical and microgeometrical deviations. Besides, in the present approach, multivariate analysis is carried out, and cutting force models are obtained in the tool and workpiece coordinate system.

Table 2 Experimental design with responses. Adapted from [55] (experimental design and $F_{a}$ ) with permission from Elsevier, license number 4153600491710

\begin{tabular}{|c|c|c|c|c|c|c|c|c|c|}
\hline \multirow[t]{2}{*}{ Std order } & \multicolumn{3}{|c|}{$\mathrm{CV}$} & \multirow{2}{*}{$\begin{array}{c}\mathrm{NV} \\
l_{t o} \\
{[\mathrm{~mm}]}\end{array}$} & \multicolumn{2}{|c|}{ Responses } & \multicolumn{3}{|c|}{ Scores } \\
\hline & $\begin{array}{c}f_{z a} \\
{[\mu \mathrm{m} / \text { tooth }]}\end{array}$ & $\begin{array}{c}f_{z t} \\
{[\mu \mathrm{m} / \text { tooth }]}\end{array}$ & $\begin{array}{c}v_{c} \\
{[\mathrm{~m} / \mathrm{min}]}\end{array}$ & & $\begin{array}{c}F_{a} \\
{[\mathrm{~N}]}\end{array}$ & $\begin{array}{c}F_{r} \\
{[\mathrm{~N}]}\end{array}$ & $P C_{1}$ & $\begin{array}{c}P C_{2} \\
- \\
\end{array}$ & $W P C_{s}$ \\
\hline 1 & 4 & 40 & 30 & 30 & 147 & 136 & -1.40 & 0.35 & -2.51 \\
\hline 2 & 11 & 40 & 30 & 30 & 249 & 248 & 0.99 & 0.10 & 1.83 \\
\hline 3 & 4 & 90 & 30 & 30 & 184 & 113 & -1.15 & -0.35 & -2.17 \\
\hline 4 & 11 & 90 & 30 & 30 & 274 & 262 & 1.44 & -0.09 & 2.63 \\
\hline 5 & 4 & 40 & 70 & 30 & 186 & 136 & -0.88 & -0.16 & -1.65 \\
\hline 6 & 11 & 40 & 70 & 30 & 263 & 294 & 1.61 & 0.36 & 3.01 \\
\hline 7 & 4 & 90 & 70 & 30 & 189 & 97 & -1.23 & -0.56 & -2.35 \\
\hline 8 & 11 & 90 & 70 & 30 & 272 & 232 & 1.14 & -0.34 & 2.02 \\
\hline 9 & 4 & 40 & 30 & 46 & 188 & 148 & -0.75 & -0.08 & -1.39 \\
\hline 10 & 11 & 40 & 30 & 46 & 266 & 284 & 1.55 & 0.22 & 2.87 \\
\hline 11 & 4 & 90 & 30 & 46 & 215 & 97 & -0.89 & -0.90 & -1.79 \\
\hline 12 & 11 & 90 & 30 & 46 & 254 & 255 & 1.12 & 0.10 & 2.08 \\
\hline 13 & 4 & 40 & 70 & 46 & 174 & 147 & -0.94 & 0.10 & -1.71 \\
\hline 14 & 11 & 40 & 70 & 46 & 266 & 253 & 1.26 & -0.07 & 2.29 \\
\hline
\end{tabular}




\begin{tabular}{cccccccccc}
15 & 4 & 90 & 70 & 46 & 198 & 91 & -1.17 & -0.74 & -2.26 \\
16 & 11 & 90 & 70 & 46 & 273 & 254 & 1.35 & -0.15 & 2.45 \\
17 & 0.5 & 65 & 50 & 38 & 30 & 25 & -3.98 & 0.80 & -7.15 \\
18 & 14.5 & 65 & 50 & 38 & 288 & 346 & 2.43 & 0.54 & 4.53 \\
19 & 7.5 & 15 & 50 & 38 & 176 & 190 & -0.50 & 0.49 & -0.83 \\
20 & 7.5 & 115 & 50 & 38 & 194 & 179 & -0.39 & 0.15 & -0.68 \\
21 & 7.5 & 65 & 10 & 38 & 171 & 202 & -0.46 & 0.67 & -0.72 \\
22 & 7.5 & 65 & 90 & 38 & 197 & 196 & -0.17 & 0.27 & -0.26 \\
23 & 7.5 & 65 & 50 & 38 & 228 & 187 & 0.14 & -0.21 & 0.22 \\
24 & 7.5 & 65 & 50 & 38 & 243 & 192 & 0.38 & -0.35 & 0.63 \\
25 & 7.5 & 65 & 50 & 38 & 227 & 202 & 0.26 & -0.05 & 0.47 \\
26 & 7.5 & 65 & 50 & 38 & 228 & 199 & 0.25 & -0.09 & 0.44 \\
\hline
\end{tabular}

\subsection{Correlation evaluation, PCA and WPC scores estimation}

The Pearson correlation coefficient between $F_{a}$ and $F_{r}$ was $r=0.83$ with $p$-value $=0.000$, confirming a significant correlation between the responses at the significance level $(\alpha)$ of 0.05 , and justifying the multivariate modelling. Considering the two responses, a spectral decomposition on the correlation matrix $\mathbf{R}$ can be performed to obtain eigenvalues and eigenvectors of $\mathbf{R}$. Thus, the results are stored in Table 3 . The first principal component accounts for 91.6\% of the variance-covariance structure between $F_{a}$ and $F_{r}$, while the second one accounts for $8.4 \%$ of the data variability. It means that $P C_{1}$ could represent the original responses since it accounts for more than $80 \%$ of the variancecovariance structure and its eigenvalue is more than 1 . However, a linear combination of both principal components may be calculated to avoid loss of information, leading to a representation of $100 \%$ of the variance-covariance structure besides solving the correlation issue. Therefore, the weighted principal component concept already stated in this work was considered for representing the uncorrelated components $P C_{1}$ and $P C_{2}$.

Table 3 Eigenanalysis of $F_{a}$ and $F_{r}$

\begin{tabular}{lll}
\hline & $P C_{1}$ & $P C_{2}$ \\
\hline Eigenvalue & 1.832 & 0.168 \\
Proportion & 0.916 & 0.084 \\
Cumulative & 0.916 & 1.000 \\
\hline Eigenvectors & $P C_{1}$ & $P C_{2}$ \\
\hline$F_{a}$ & 0.707 & -0.707 \\
$F_{r}$ & 0.707 & 0.707 \\
\hline
\end{tabular}

To illustrate the original responses and their principal components, Fig. 6 shows a scatter plot of the original responses correlated positively. Graphically, it can be illustrated in Fig. 6 what was stated in the previous paragraph in relation to the data variability explained by $P C_{1}$ and $P C_{2}$. In Fig. 6, it is seen that the variability explained by $P C_{2}$, inherent to the $P C_{2}$ axe, is lower than the variability explained by $P C_{1}$, inherent to $P C_{1}$ axe. Although $P C_{1}$ explains most of the variance-covariance structure, $P C_{2}$ should not be ignored when desiring to totally explain the variance-covariance structure inherent to $F_{a}$ and $F_{r}$; therefore, it favors using the weighted principal component technique.

In Fig. 6, the ellipse is expressed by $(\mathbf{y}-\overline{\mathbf{y}})^{T} \mathbf{S}^{-1}(\mathbf{y}-\overline{\mathbf{y}})=c^{2}$, where $\mathbf{y}^{T}=\left(F_{a}, F_{r}\right)$ is the vector of the original outcomes, $\overline{\mathbf{y}}^{T}=\left(\bar{F}_{a} ; \bar{F}_{r}\right)=(214.61 ; 190.96), c$ is a constant and in this work $c^{2}=2.45$, which represents an ellipse at a confidence level of $95 \%$, and

$\mathbf{S}=\left[\begin{array}{ll}2998.57 & 3370.14 \\ 3370.14 & 5473.97\end{array}\right]$

is the estimated variance-covariance matrix whose main diagonal contains the variances of $F_{a}$ and $F_{r}$, and the secondary diagonal contains the covariance between $F_{a}$ and $F_{r}$. There is a single point outside the confidence ellipse, which is correspondent to $F_{a}=30 \mathrm{~N}$ and $F_{r}=25 \mathrm{~N}$, according to Table 2, row 17. In Fig. 7, it is seen that through the Mahalanobis distance approach, there is one outlier between the values of the outcomes $F_{a}$ and $F_{r}$, and this outlier is related to the point outside the confidence ellipse in Fig. 6 . This point is inherent to $f_{z a}$ set to $0.5 \mu \mathrm{m} /$ tooth, which is its positive axial point. In the central composite design, the factorial and axial points are at the same and highest distance from the center and, therefore, present the highest variance levels regarding the experimental region. As $f_{z a}$ is generally 
the most influent variable on cutting force components, it is expected this variability in extreme points. To assure a balanced design and sufficient degrees of freedom, this point is important to estimate the quadratic effect of $f_{z a}$.

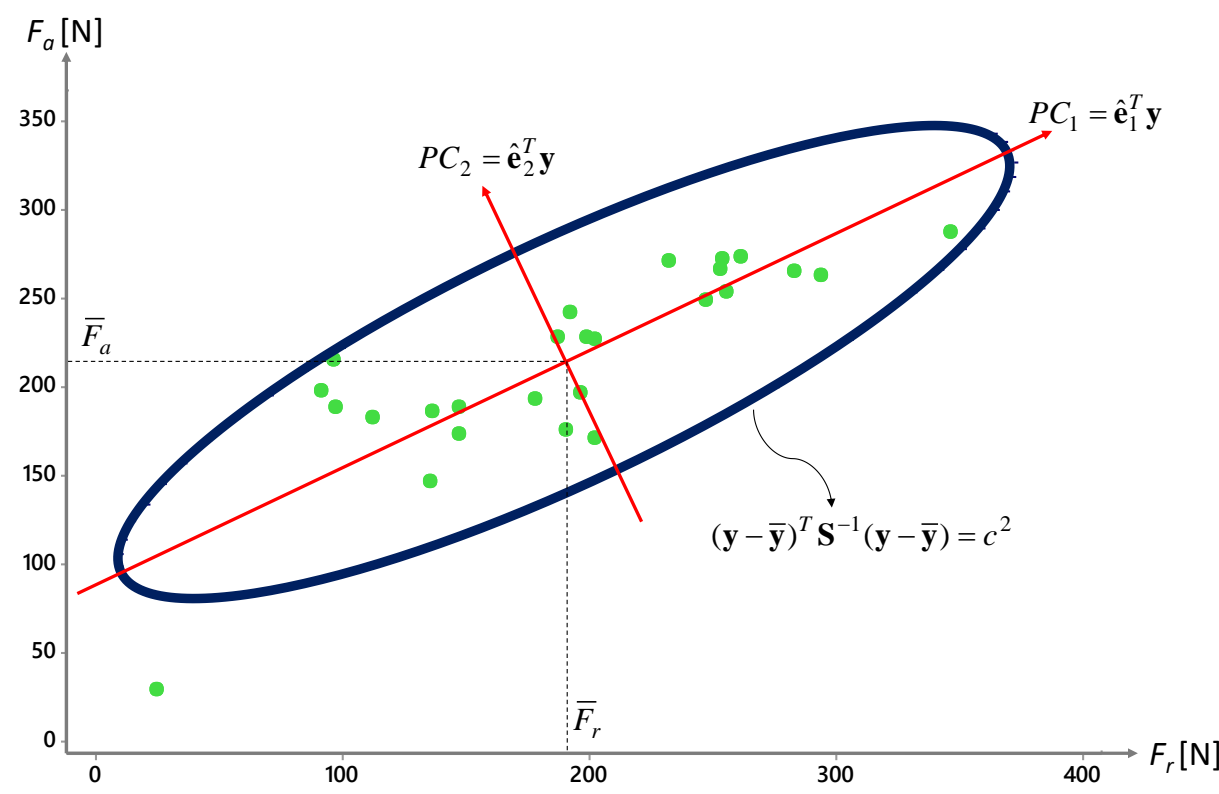

Fig. 6 Correlated original responses $(r=0.832)$

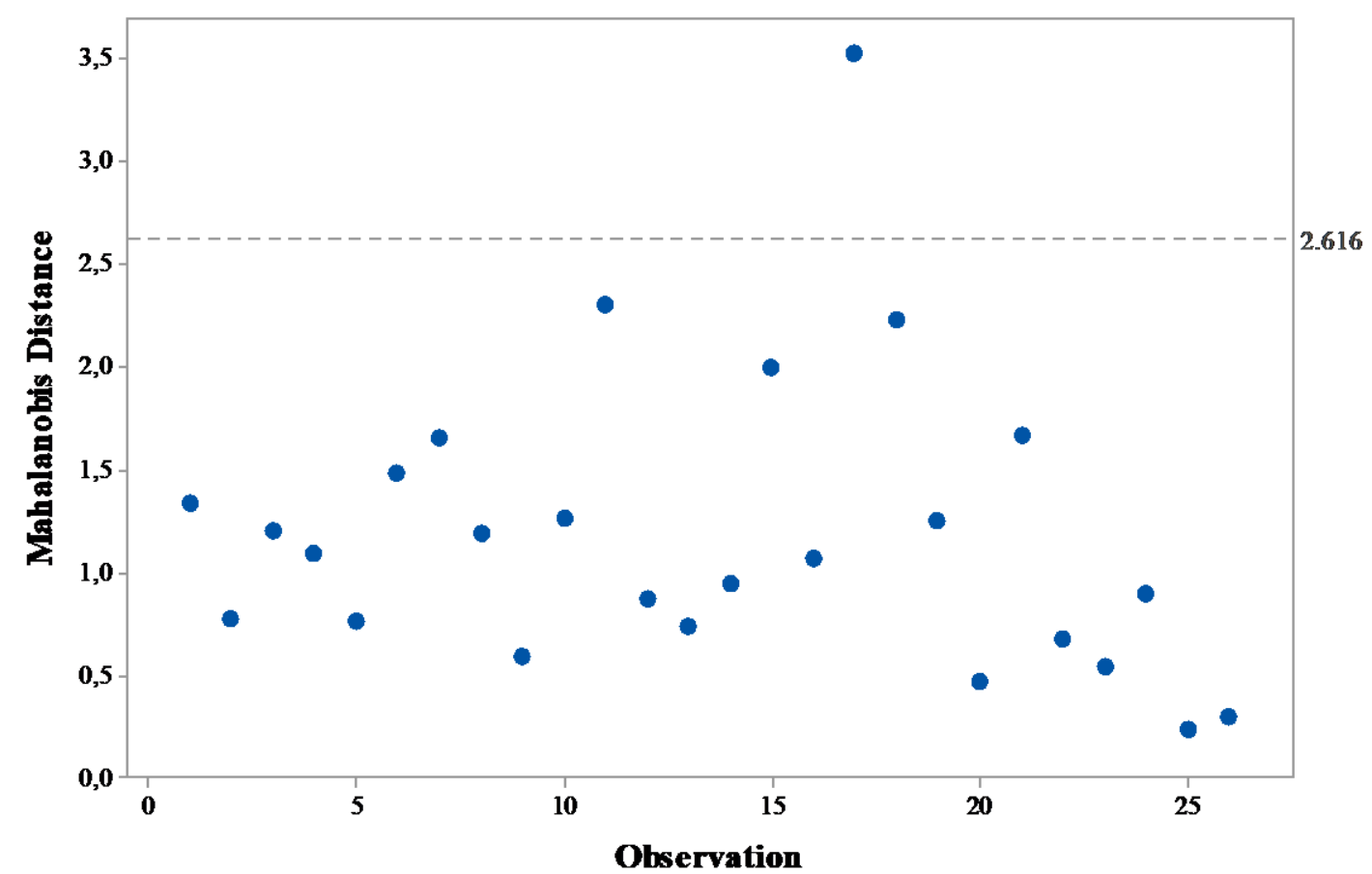

Fig. 7 Mahalanobis distance measures

The observed uncorrelation considering the new coordinate system with $P C_{l}$ and $P C_{2}$ axes is quite important for Step 5 so that the coefficients of the $W P C(\mathbf{x}, \mathbf{z})$ response surface model can be correctly estimated.

The scores of the weighted principal component, which represent the correlated responses without loss of information, were calculated through linear combination as in Eq. (16), considering the two principal component scores and their respective eigenvalues. The scores of the weighted principal component were stored in Table 2. 


\subsection{Response surface model and RPD for WPC, $F_{a}$ and $F_{r}$}

Henceforth, the variables will be presented in coded units; therefore, the response surface models will be also presented in coded units unless it is written uncoded units. The full quadratic $\operatorname{WPC}(\mathbf{x}, \mathbf{z})$ response surface model proposed in Eq. (21) was calculated using the WLS algorithm and is expressed in Eq. (30). The full quadratic response surface models $F_{a}(\mathbf{x}, \mathbf{z})$ and $F_{r}(\mathbf{x}, \mathbf{z})$ were also calculated and are expressed in Eq. (31) and Eq. (32), respectively. The quadratic regression models represented by Eq. (31) and Eq. (32) are essential to apply the Pareto optimal solutions considering the results of the $\mathrm{MMSE}_{\mathrm{W}} \mathrm{NBI}$ optimization in the original responses. The statistical summary of the regression models, presented in Table 4, confirms good adjustments of data and no lack-of-fit (LOF) for all the full quadratic response surface models at the significance level of 0.05 . The $W P C(\mathbf{x}, \mathbf{z})$ model presented good determination coefficients, confirming high data variability explanation, with $R_{a d j}^{2}=95.11 \%$, and suitable properties of prediction of the response model, $R_{\text {pred }}^{2}=81.73 \%$. Furthermore, the models of the original responses also presented good determination coefficients and properties of prediction.

$$
\begin{aligned}
& W P C(\mathbf{x}, \mathbf{z})=0.605+2.231 f_{z a}-0.074 f_{z t}+0.051 v_{c}+0.100 l_{t o}-0.087 f_{z a}^{2}-0.255 f_{z t}^{2}-0.193 v_{c}^{2}+0.053 f_{z a} \times f_{z t} \\
& +0.046 f_{z a} \times v_{c}-0.094 f_{z a} \times l_{t o}-0.139 f_{z t} \times v_{c}-0.055 f_{z t} \times l_{t o}-0.147 v_{c} \times l_{t o} \\
& F_{a}(\mathbf{x}, \mathbf{z})=242.575+43.100 f_{z a}+7.049 f_{z t}+2.905 v_{c}+5.151 l_{t o}-9.472 f_{z a}^{2}-7.663 f_{z t}^{2}-7.882 v_{c}^{2}-6.001 f_{z a} \times f_{z t} \\
& +2.000 f_{z a} \times v_{c}-4.612 f_{z a} \times l_{t o}+0.393 f_{z t} \times v_{c}-3.480 f_{z t} \times l_{t o}-1.946 v_{c} \times l_{t o} \\
& F_{r}(\mathbf{x}, \mathbf{z})=194.422+72.028 f_{z a}-12.407 f_{z t}-1.429 v_{c}+1.787 l_{t o}-2.059 f_{z a}^{2}-2.134 f_{z t}^{2}+1.205 v_{c}^{2}+7.009 f_{z a} \times f_{z t} \\
& +0.973 f_{z a} \times v_{c}+1.490 f_{z a} \times l_{t o}-3.479 f_{z t} \times v_{c}-3.223 f_{z t} \times l_{t o}-0.070 v_{c} \times l_{t o}
\end{aligned}
$$

Table 4 Model summary

\begin{tabular}{llll}
\hline Statistics & $F_{a}$ & $F_{r}$ & $W P C$ \\
\hline$\hat{\sigma}^{2}$ & 1.11 & 1.40 & 1.30 \\
$R^{2}(\%)$ & 98.33 & 99.98 & 97.65 \\
$R^{2}{ }_{\text {adj }}(\%)$ & 96.52 & 99.95 & 95.11 \\
$R_{\text {pred }}(\%)$ & 83.79 & 98.57 & 81.73 \\
LOF (p-value) & 0.42 & 0.53 & 0.17 \\
\hline
\end{tabular}




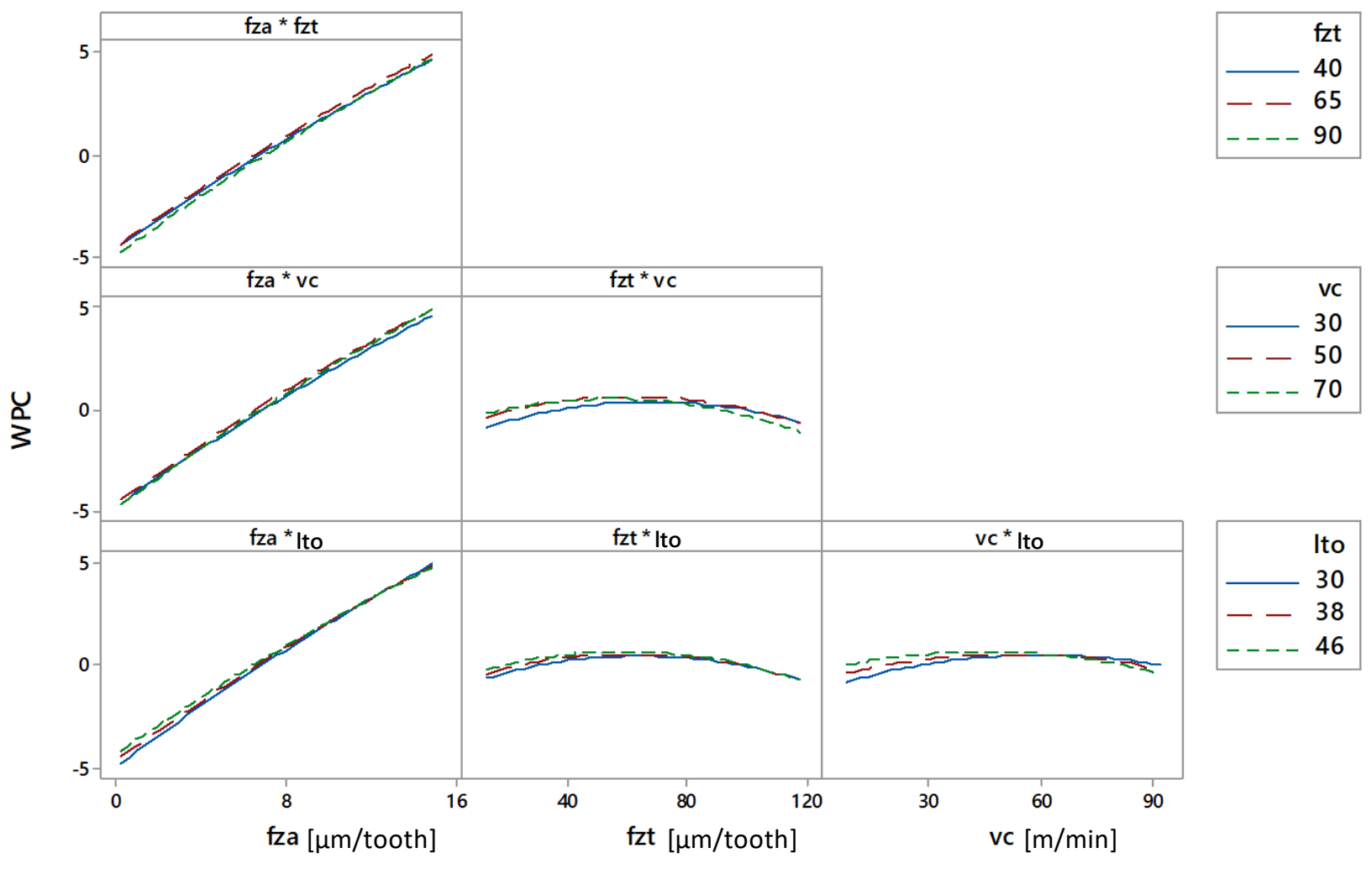

Fig. 8 Interaction plot for $W P C(\mathbf{x}, \mathbf{z})$ response surface model

Fig. 8 shows interaction plots for $\operatorname{WPC}(\mathbf{x}, \mathbf{z})$. In these plots, process and noise effects on the cutting forces are illustrated. The positive effect of $f_{z a}$ on all the response surface models is remarkable. In this line of reasoning, the axial feed per tooth, which generates continuous cut, has a significant role on the cutting force levels.

The literature assures that the effect of $f_{z a}$ is positive and remarkable on the cutting forces components because as $f_{z a}$ increases with $f_{z t}$ kept unchanged, the axial cutting depth $a_{p}{ }^{*}$ increases, and both the areas that are cut by peripheral and frontal cutting edges increase. However, the low effect of $f_{z t}$ was observed because when $f_{z t}$ increases with $f_{z a}$ kept constant, the chip thickness in the peripheral cut increases, but the axial cutting depth $a_{p}{ }^{*}$ decreases [56].

The interaction plot between $v_{c}$ and $l_{t o}$ shows that cutting velocity levels between 60 and $70 \mathrm{~m} / \mathrm{min}$ are insensitive to tool overhang length variation. When performing the optimization, these levels of cutting velocity are confirmed. It means that with these levels of cutting velocity, the cutting force components will not vary due to the tool overhang length variation. Then, according to the geometry of the workpiece, the experimenter may set the necessary tool overhang length without affecting the cutting forces during helical milling.

As graphically illustrated, no significant linear effect is present regarding $f_{z t}$ and $v_{c}$. However, some curvature may be observed in these parameters in relation to cutting forces. Besides, the slope difference in the interactions may guide the search for robustness. For instance, in the interaction between $v_{c}$ and $l_{t o}$, cutting velocity levels close to $60 \mathrm{~m} / \mathrm{min}$ make the process robust to the tool overhang length variation. It means that with this cutting velocity, the cutting forces will not be affected by the variation of the tool overhang length.

Through the RPD approach, as proposed in Eq. (22) and Eq. (23), mean and variance equations were obtained in function of controllable variables, considering the variation transmitted by the noise factor. These models are robust to the tool overhang length variation. The coefficients of the mean and variance equations of $W P C(\mathbf{x}, \mathbf{z}), F_{a}(\mathbf{x}, \mathbf{z})$ and $F_{r}(\mathbf{x}, \mathbf{z})$ are in Eqs. (33-38).

Multivariate robust optimal setting for the machining force components in the helical milling process must be obtained by minimizing the mean and variance fitted equations of $\operatorname{WPC}(\mathbf{x}, \mathbf{z})$. The achieved optimal levels of the controllable variables must optimize the original correlated responses $F_{a}$ and $F_{r}$, minimizing the influence of the tool overhang length variation and considering the influence of the variance-covariance structure in the modelling and optimization problem. Fig. 9 shows the response surface plots for the mean and variance of WPC $(\mathbf{x}, \mathbf{z})$ under different perspectives. 

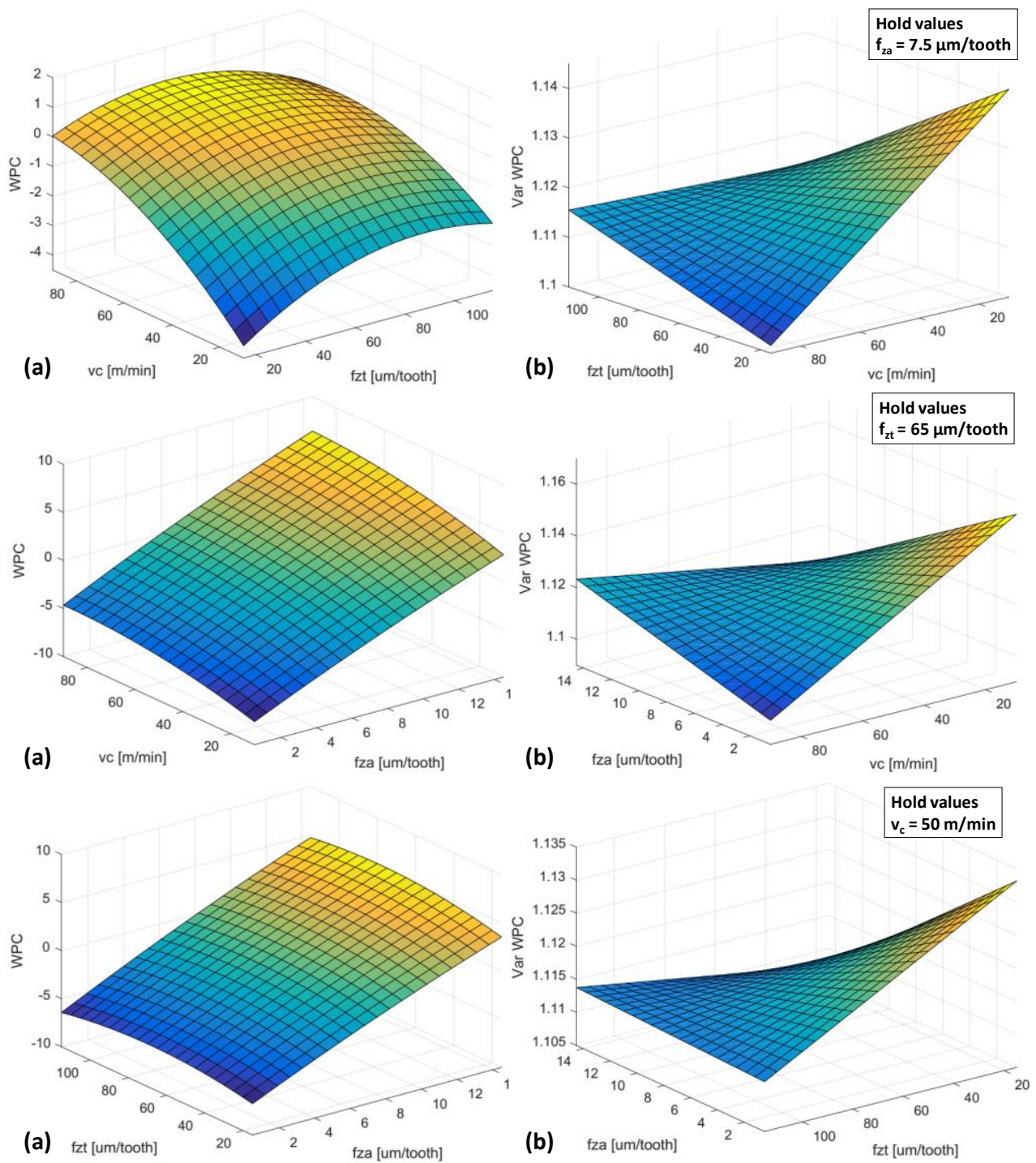

Fig. 9 Surface plots for (a) WPC mean model and (b) $W P C$ variance model

$$
\begin{aligned}
& E_{z}[W P C(\mathbf{x}, \mathbf{z})]=0.605+2.231 f_{z a}-0.074 f_{z t}+0.051 v_{c}-0.087 f_{z a}^{2}-0.255 f_{z t}^{2}-0.193 v_{c}^{2}+0.053 f_{z a} \times f_{z t} \\
& +0.046 f_{z a} \times v_{c}-0.139 f_{z t} \times v_{c} \\
& V_{z}[W P C(\mathbf{x}, \mathbf{z})]=1.307-0.019 f_{z a}-0.011 f_{z t}-0.029 v_{c}+0.009 f_{z a}^{2}+0.003 f_{z t}^{2}+0.022 v_{c}^{2}+0.010 f_{z a} \times f_{z t} \\
& +0.028 f_{z a} \times v_{c}+0.016 f_{z t} \times v_{c} \\
& E_{z}\left[F_{a}(\mathbf{x}, \mathbf{z})\right]=242.575+43.100 f_{z a}+7.049 f_{z t}+2.905 v_{c}-9.472 f_{z a}^{2}-7.663 f_{z t}^{2}-7.882 v_{c}^{2}-6.001 f_{z a} \times f_{z t} \\
& +2.000 f_{z a} \times v_{c}+0.393 f_{z t} \times v_{c} \\
& V_{z}\left[F_{a}(\mathbf{x}, \mathbf{z})\right]=27.642-47.511 f_{z a}-35.850 f_{z t}-20.050 v_{c}+21.271 f_{z a}^{2}+12.110 f_{z t}^{2}+3.788 v_{c}^{2}+32.100 f_{z a} \times f_{z t} \\
& +17.953 f_{z a} \times v_{c}+13.546 f_{z t} \times v_{c} \\
& E_{z}\left[F_{r}(\mathbf{x}, \mathbf{z})\right]=194.422+72.028 f_{z a}-12.407 f_{z t}-1.429 v_{c}-2.059 f_{z a}^{2}-2.134 f_{z t}^{2}+1.205 v_{c}^{2}+7.009 f_{z a} \times f_{z t} \\
& +0.973 f_{z a} \times v_{c}-3.479 f_{z t} \times v_{c} \\
& V_{z}\left[F_{r}(\mathbf{x}, \mathbf{z})\right]=4.594+5.327 f_{z a}-11.521 f_{z t}-0.249 v_{c}+2.221 f_{z a}^{2}+10.388 f_{z t}^{2}+0.005 v_{c}^{2}-9.606 f_{z a} \times f_{z t} \\
& -0.208 f_{z a} \times v_{c}+0.449 f_{z t} \times v_{c}
\end{aligned}
$$




\subsection{Utopia, nadir and payoff matrix calculation for multi-objective optimization}

For any multi-objective optimization procedure, it is necessary to calculate the utopia and pseudo nadir for each response, besides the payoff matrix. The utopia points

$E_{z}^{*}\left[\mathbf{x}_{E_{z}(W P C)}^{*}\right]=E_{z}^{*}(-1.67 ; 0.16 ; 0.04)=-3.406$ and

$V_{z}^{*}\left[\mathbf{x}_{V_{z}(W P C)}^{*}\right]=V_{z}^{*}(0.28 ; 0.16 ; 0.44)=1.297$

were obtained as solutions of the non-linear optimization problems $\operatorname{Min}_{\mathbf{x}^{T} \mathbf{x} \leq 2.828}\left\{E_{z}[W P C(\mathbf{x}, \mathbf{z})]\right\}$ and $\operatorname{Min}_{\mathbf{x}^{T} \mathbf{x} \leq 2.828}\left\{V_{z}[W P C(\mathbf{x}, \mathbf{z})]\right\}$, respectively. Note that $W P C$ can assume negative values because it is standardized and dimensionless. The uncoded levels of the utopia points are $E_{z}^{*}(1.6 \mu \mathrm{m} /$ tooth $; 69.1 \mu \mathrm{m} /$ tooth $; 50.7 \mathrm{~m} / \mathrm{min})$ and $V_{z}^{*}(8.5 \mu \mathrm{m} /$ tooth $; 69.0 \mu \mathrm{m} /$ tooth $; 58.8 \mathrm{~m} / \mathrm{min})$.

The optimal vector for $E_{z}[W P C(\mathbf{x}, \mathbf{z})]$ combines the lowest level of $f_{z a}=1.6 \mu \mathrm{m} /$ tooth with $f_{z t}=69.1 \mu \mathrm{m} /$ tooth slightly larger than the center point $(65.0 \mu \mathrm{m} /$ tooth $)$, and $v_{c}=50.7 \mathrm{~m} / \mathrm{min}$ is practically on center point $(50.0 \mathrm{~m} / \mathrm{min})$. The optimal levels, which minimizes the variance of WPC due to the tool overhang length effect, presents levels of the controllable factors higher than their center points, with $f_{z a}=8.5 \mu \mathrm{m} /$ tooth (center point equals to $7.5 \mu \mathrm{m} /$ tooth) and $f_{z t}=$ $69.0 \mu \mathrm{m} /$ tooth close of the center point level $(65.0 \mu \mathrm{m} /$ tooth $)$, and $v_{c}=58.8 \mathrm{~m} / \mathrm{min}$, which is between its center point $(50.0 \mathrm{~m} / \mathrm{min})$ and its upper factorial level $(70.0 \mathrm{~m} / \mathrm{min})$.

The payoff matrix is built using the respective optimal factors levels for $E_{z}[W P C(\mathbf{x}, \mathbf{z})]$ and $V_{z}[W P C(\mathbf{x}, \mathbf{z})]$, i.e., $(-1.67 ; 0.16 ; 0.04)$ and $(0.28 ; 0.16 ; 0.44)$. The pseudo nadir points

$E_{z}^{P N}\left[\mathbf{x}_{V_{z}(W P C)}^{*}\right]=E_{z}^{P N}(0.28 ; 0.16 ; 0.44)=1.187$, and

$V_{z}^{P N}\left[\mathbf{x}_{E_{z}(W P C)}^{*}\right]=V_{z}^{P N}(-1.67 ; 0.16 ; 0.04)=1.356$

were obtained, which makes it possible to build the payoff matrix $\boldsymbol{\Phi}$ as

$\boldsymbol{\Phi}=\left[\begin{array}{cc}-3.406 & 1.187 \\ 1.356 & 1.297\end{array}\right]$

\subsection{MMSEw-NBI multi-objective optimization}

Applying the MMSE $\mathrm{W}_{\mathrm{W}} \mathrm{NBI}$ method iteratively, the optimization results were achieved and are resumed in Table 5. The Pareto frontier considering $E_{z}[\operatorname{WPC}(\mathbf{x}, \mathbf{z})]=E_{z}(\mathbf{x})$ and $V_{z}[W P C(\mathbf{x}, \mathbf{z})]=V_{z}(\mathbf{x})$ is exposed in Fig. 10(a). For comparison purposes, the MMSE $\mathrm{w}_{\mathrm{w}}$, Eq. (24), was solved iteratively and the Pareto frontier is exposed in Fig. 10(b). The MMSE $\mathrm{w}_{\mathrm{w}}$ did not generate an equispaced frontier. On the other hand, the proposed MMSE $\mathrm{W}_{-\mathrm{NBI}}$ method was efficient in generating equispaced Pareto optimal solutions. It is important to use a Pareto filter to exclude the dominated points from the optimization results. In this study, the MMSE ${ }_{W}-\mathrm{NBI}$ method generated four dominated solutions.

The derived results for the mean and variance of $F_{a}$ and $F_{r}$, are also resumed in Table 5. It is relevant to state that related to $F_{a}$, there were higher levels of Pareto optimal results in terms of mean and variance when compared to the mean and variance levels of $F_{r}$. The Pareto optimal results of variance for $F_{r}$ presented low dispersion. In Table 5, standard deviation (SD) results were also stored since in manufacturing situations this estimate is more useful than the variance estimate. 

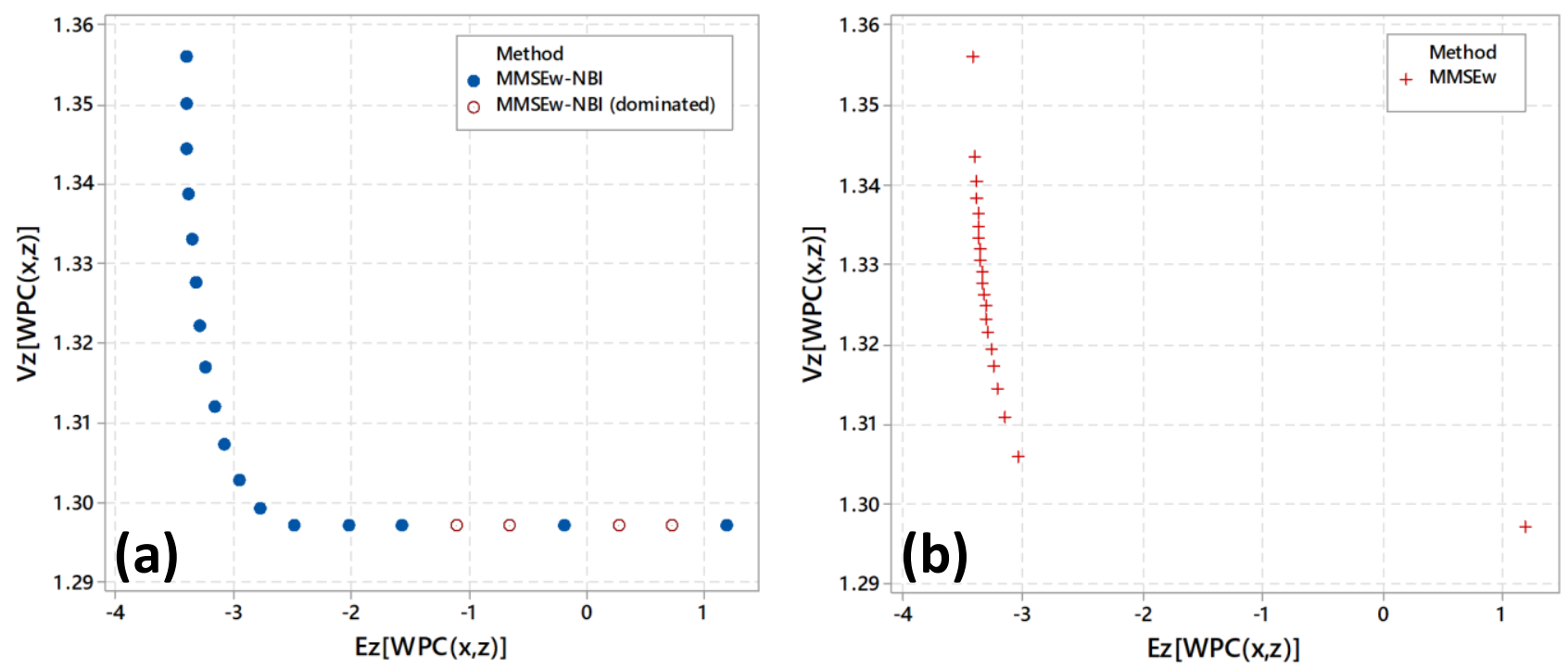

Fig. 10 Pareto frontiers of (a) MMSE $E_{\mathrm{w}}-\mathrm{NBI}$ method and (b) $\mathrm{MMSE}_{\mathrm{w}}$ method 


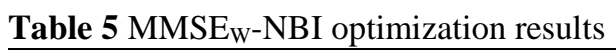

\begin{tabular}{|c|c|c|c|c|c|c|c|c|c|c|c|c|c|c|}
\hline \multirow{4}{*}{$w$} & \multicolumn{6}{|c|}{ Process variables } & \multicolumn{8}{|c|}{ Responses } \\
\hline & \multicolumn{3}{|c|}{ coded } & \multicolumn{3}{|c|}{ Uncoded } & \multirow{2}{*}{$E_{z}\left[F_{a}\right]$} & \multirow{2}{*}{$\operatorname{Var}_{z}\left[F_{a}\right]$} & \multirow{2}{*}{$S D_{z}\left[F_{a}\right]$} & \multirow{2}{*}{$E_{z}\left[F_{r}\right]$} & \multirow{2}{*}{$\operatorname{Var}_{z}\left[F_{r}\right]$} & \multirow{2}{*}{$S D_{z}\left[F_{r}\right]$} & \multirow{3}{*}{$E_{z}[W P C]$} & \multirow{3}{*}{$\operatorname{Var}_{z}[\mathrm{WPC}$} \\
\hline & \multirow{2}{*}{$f_{z a}$} & \multirow{2}{*}{$f_{z t}$} & \multirow{2}{*}{$v_{c}$} & $f_{z a}$ & $f_{z t}$ & $v_{c}$ & & & & & & & & \\
\hline & & & & {$[\mu \mathrm{m} /$ tooth $]$} & {$[\mu \mathrm{m} /$ tooth $]$} & {$[\mathrm{m} / \mathrm{min}]$} & {$[\mathrm{N}]$} & {$\left[\mathrm{N}^{2}\right]$} & {$[\mathrm{N}]$} & {$[\mathrm{N}]$} & {$\left[\mathrm{N}^{2}\right]$} & {$[\mathrm{N}]$} & & \\
\hline 0 & 0.28 & 0.16 & 0.44 & 8.5 & 69.0 & 58.8 & 254.6 & 7.1 & 2.7 & 212.2 & 4.2 & 2.0 & 1.187 & 1.297 \\
\hline $0.05 *$ & 0.09 & 0.21 & 0.54 & 7.8 & 70.3 & 60.9 & 246.7 & 9.8 & 3.1 & 197.3 & 2.8 & 1.7 & 0.728 & 1.297 \\
\hline $0.1^{*}$ & -0.10 & 0.26 & 0.64 & 7.2 & 71.6 & 62.9 & 238.2 & 12.9 & 3.6 & 182.5 & 2.0 & 1.4 & 0.269 & 1.297 \\
\hline 0.15 & -0.28 & 0.32 & 0.74 & 6.5 & 73.0 & 64.8 & 229.3 & 16.2 & 4.0 & 167.9 & 1.5 & 1.2 & -0.191 & 1.297 \\
\hline $0.2 *$ & -0.46 & 0.36 & 0.84 & 5.9 & 74.1 & 66.7 & 219.8 & 20.2 & 4.5 & 153.5 & 1.4 & 1.2 & -0.650 & 1.297 \\
\hline $0.25 *$ & -0.63 & 0.40 & 0.93 & 5.3 & 75.0 & 68.6 & 209.8 & 24.6 & 5.0 & 139.3 & 1.6 & 1.3 & -1.109 & 1.297 \\
\hline 0.3 & -0.80 & 0.41 & 1.03 & 4.7 & 75.4 & 70.7 & 199.0 & 29.9 & 5.5 & 125.5 & 2.1 & 1.4 & -1.569 & 1.297 \\
\hline 0.35 & -0.96 & 0.47 & 1.11 & 4.2 & 76.9 & 72.3 & 188.6 & 34.1 & 5.8 & 111.2 & 2.9 & 1.7 & -2.028 & 1.297 \\
\hline 0.4 & -1.10 & 0.68 & 1.08 & 3.7 & 82.0 & 71.5 & 181.4 & 34.3 & 5.9 & 94.3 & 5.9 & 2.4 & -2.483 & 1.297 \\
\hline 0.45 & -1.26 & 0.62 & 0.93 & 3.1 & 80.6 & 68.6 & 173.4 & 49.7 & 7.0 & 83.2 & 6.1 & 2.5 & -2.774 & 1.299 \\
\hline 0.5 & -1.36 & 0.57 & 0.81 & 2.7 & 79.3 & 66.2 & 167.9 & 62.6 & 7.9 & 76.6 & 6.0 & 2.4 & -2.950 & 1.303 \\
\hline 0.55 & -1.43 & 0.52 & 0.71 & 2.5 & 78.1 & 64.2 & 163.8 & 74.0 & 8.6 & 72.1 & 5.7 & 2.4 & -3.073 & 1.307 \\
\hline 0.6 & -1.49 & 0.48 & 0.62 & 2.3 & 77.0 & 62.4 & 160.4 & 84.6 & 9.2 & 68.9 & 5.5 & 2.3 & -3.164 & 1.312 \\
\hline 0.65 & -1.53 & 0.44 & 0.54 & 2.1 & 75.9 & 60.7 & 157.6 & 94.4 & 9.7 & 66.6 & 5.2 & 2.3 & -3.234 & 1.317 \\
\hline 0.7 & -1.57 & 0.40 & 0.46 & 2.0 & 74.9 & 59.1 & 155.2 & 103.6 & 10.2 & 65.0 & 4.9 & 2.2 & -3.287 & 1.322 \\
\hline 0.75 & -1.60 & 0.36 & 0.38 & 1.9 & 73.9 & 57.6 & 153.2 & 112.3 & 10.6 & 63.9 & 4.5 & 2.1 & -3.328 & 1.328 \\
\hline 0.8 & -1.62 & 0.32 & 0.31 & 1.8 & 72.9 & 56.1 & 151.4 & 120.7 & 11.0 & 63.2 & 4.2 & 2.0 & -3.359 & 1.333 \\
\hline 0.85 & -1.64 & 0.28 & 0.24 & 1.8 & 72.0 & 54.7 & 149.9 & 128.6 & 11.3 & 62.9 & 3.9 & 2.0 & -3.381 & 1.339 \\
\hline 0.9 & -1.66 & 0.24 & 0.17 & 1.7 & 71.0 & 53.4 & 148.6 & 136.4 & 11.7 & 63.0 & 3.5 & 1.9 & -3.396 & 1.344 \\
\hline 0.95 & -1.67 & 0.20 & 0.10 & 1.7 & 70.1 & 52.0 & 147.5 & 143.6 & 12.0 & 63.3 & 3.2 & 1.8 & -3.404 & 1.350 \\
\hline 1 & -1.67 & 0.16 & 0.04 & 1.6 & 69.1 & 50.7 & 146.5 & 150.6 & 12.3 & 64.0 & 2.9 & 1.7 & -3.406 & 1.356 \\
\hline \multicolumn{7}{|c|}{ *dominated solutions } & & & & & & & & \\
\hline
\end{tabular}


In Table 5, it is observed that for higher levels of the axial feed per tooth, the expected values for the machining forces are also higher. On the other hand, for lower levels of the axial feed per tooth, the expected values for the machining forces are lower as well. It can be explained by considering the technological characteristic of the helical milling represented by its kinematics and by the two points in Table 5, the points inherent to $w=0$ and $w=1$, which has similar levels for the tangential feed per tooth. In this way, the points are $\mathbf{x}_{w=0}^{*}=[8.5 \mu \mathrm{m} /$ tooth; $69 \mu \mathrm{m} /$ tooth; 58.8 $\mathrm{m} / \mathrm{min}]$ and $\mathbf{x}_{w=1}^{*}=[1.6 \mu \mathrm{m} /$ tooth; $69.1 \mu \mathrm{m} /$ tooth; $50.7 \mathrm{~m} / \mathrm{min}]$. These points were chosen because they present a great discrepancy between the levels of $f_{z a}$ and because this factor is the most significant one for both machining forces. In this line of reasoning, the first point, $\mathbf{x}_{w=0}^{*}$, has higher levels of the machining forces if compared to the levels of the forces related to the second point, $\mathbf{x}_{w=1}^{*}$, because the axial cutting depth related to $\mathbf{x}_{w=0}^{*}=[8.5 ; 69 ; 58.8]$, based on Eq. (5), is higher than the axial cutting depth related to $\mathbf{x}_{w=1}^{*}=[1.6 ; 69.1 ; 50.7]$. It is easy to demonstrate this finding through the analysis of the helical milling kinematics. First, through Eq. (1-4), some helical milling parameters were evaluated. Without loss of generality, they are presented in Table (6).

Table 6 - Helical milling parameters for $\mathbf{x}_{w=0}^{*}$ and $\mathbf{x}_{w=1}^{*}$

\begin{tabular}{lccc}
\hline Parameters & $\mathbf{x}_{w=0}^{*}$ & $\mathbf{x}_{w=1}^{*}$ & Unit \\
\hline$f_{z a}$ & 0.0085 & 0.0016 & $\mathrm{~mm}$ \\
$f_{z t}$ & 0.0690 & 0.0691 & $\mathrm{~mm}$ \\
$v_{c}$ & 58.8 & 50.7 & $\mathrm{~m} / \mathrm{min}$ \\
\hline$v_{f h a}$ & 34.0 & 6.4 & $\mathrm{~mm} / \mathrm{min}$ \\
$v_{f t}$ & 276.0 & 276.4 & $\mathrm{~mm} / \mathrm{min}$ \\
$v_{f h t}$ & 92.0 & 92.1 & $\mathrm{~mm} / \mathrm{min}$ \\
$v_{f}$ & 98.1 & 92.4 & $\mathrm{~mm} / \mathrm{min}$ \\
\hline
\end{tabular}

The values in Table (6) are important for the calculation of the angle of the helix, Eq. (6). For $\mathbf{x}_{w=0}^{*}=[8.5 ; 69$; 58.8], the angle of the helix is

$\alpha\left[\mathbf{x}_{w=0}^{*}\right]=\arctan \left(\frac{34.0}{92.0}\right)=0.354$ radian

and for $\mathbf{x}_{w=1}^{*}=[1.6 ; 69.1 ; 50.7]$, the angle of the helix is

$\alpha\left[\mathbf{x}_{w=1}^{*}\right]=\arctan \left(\frac{6.4}{92.1}\right)=0.069$ radian

By analyzing the angle of the helix related to these two points, it is already easy to realize that due to the higher level of $f_{z a}$ in $\mathbf{x}_{w=0}^{*}$, higher cutting efforts are demanded since $f_{z a}$ contributes to the increase of the axial feed velocity of the helix $\left(v_{f h a}\right)$, increasing the angle of the helix, see Fig. 1 , and because $f_{z a}$ has a high impact on the cutting forces. To support this statement, the axial cutting depth were also evaluated for better understating these higher efforts requirement. For $\mathbf{x}_{w=0}^{*}=[8.5 ; 69 ; 58.8]$, the axial cutting depth, based on Eq. (5), is

$a_{p}^{*}\left[\mathbf{x}_{w=0}^{*}\right]=\tan (0.354) \cdot \pi \cdot 5=5.8 \mathrm{~mm}$

In contrast, for $\mathbf{x}_{w=1}^{*}=[1.6 ; 69.1 ; 50.7]$, the axial cutting depth is

$a_{p}^{*}\left[\mathbf{x}_{w=1}^{*}\right]=\tan (0.069) \cdot \pi \cdot 5=1.1 \mathrm{~mm}$ 
As it is observed, when $f_{z a}$ increases, the axial cutting depth increases, both areas that are cut by peripheral and frontal cutting edges also increase, and it requires high cutting efforts, resulting in higher levels of machining forces.

\subsection{Confirmation runs}

Confirmation runs were carried out on the robust Pareto optimal vector $\mathbf{x}_{w=0.5}^{*}=[-1.36 ; 0.57 ; 0.81]$, which in decoded units stands for $\mathbf{x}_{w=0.5}^{*}=[2.7 \mu \mathrm{m} /$ tooth; $79.3 \mu \mathrm{m} /$ tooth; $66.2 \mathrm{~m} / \mathrm{min}]$. The weight $w=0.5$ was chosen to guarantee a good compromise between mean and variance. This Pareto optimal vector resulted in $E_{z}[W P C]=-2.950, \operatorname{Var}_{z}[W P C]=1.303$, $E_{z}\left[F_{a}\right]=167.9 \mathrm{~N}, S D\left[F_{a}\right]=7.9 \mathrm{~N}, E_{z}\left[F_{r}\right]=76.6 \mathrm{~N}, S D\left[F_{r}\right]=2.4 \mathrm{~N}$. The power curve in Fig. 11 guarantees that a sample with $n=3$ helical milling confirmation tests is sufficient to detect a difference of $5 \mathrm{~N}$ in $F_{a}$ and $F_{r}$, considering their respective experimental standard deviations, with probabilities to detect this difference equal to 0.98 and 0.95 , respectively.
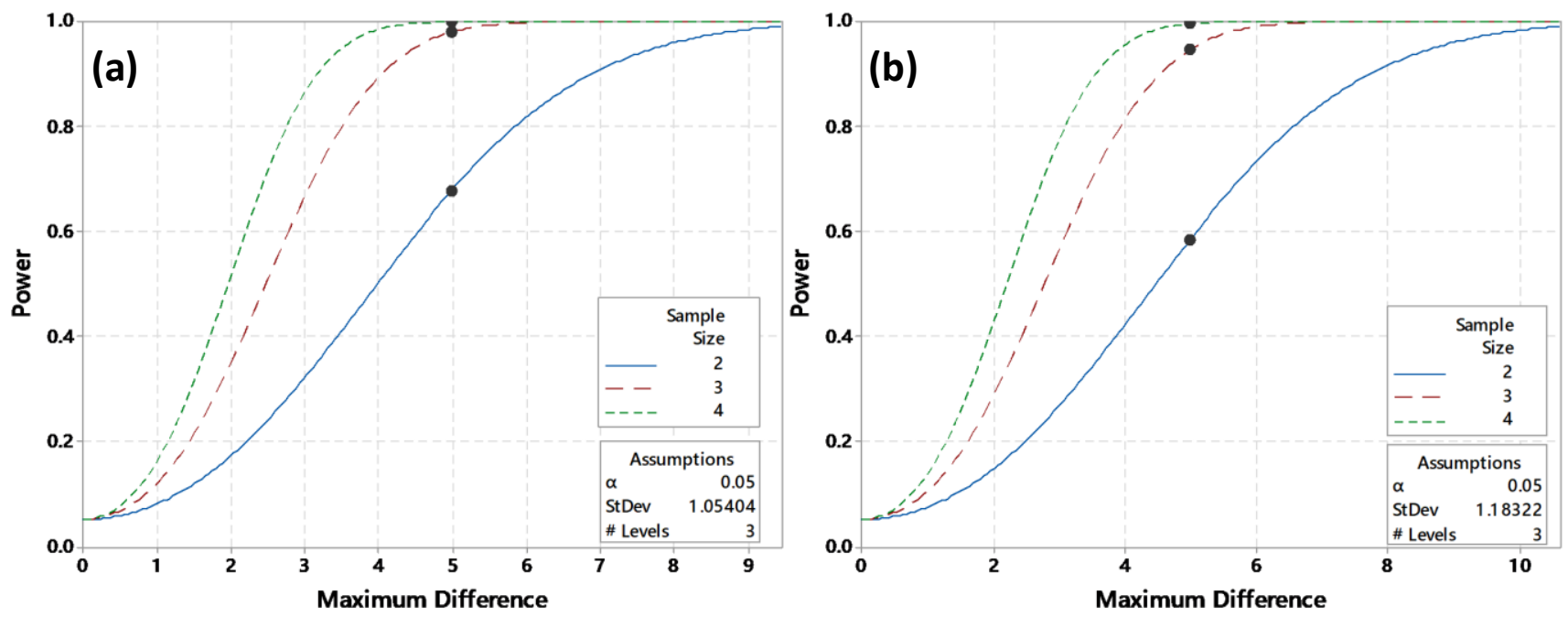

Fig. 11 Power curve for (a) $F_{a}$ and for (b) $F_{r}$

Table 7 presents the results of the confirmation runs considering the three $l_{t o}$ levels and three replications, as determined by the power test. The mean and standard deviation levels obtained for $F_{a}$ and $F_{r}$ were $E_{z}\left[F_{a}\right]=167.9 \mathrm{~N}$, with $S D_{z}\left[F_{a}\right]=7.9, E_{z}\left[F_{r}\right]=76.6 \mathrm{~N}$, with $S D_{z}\left[F_{r}\right]=2.4 \mathrm{~N}$. The Pearson correlation test for $F_{a}$ and $F_{r}$ resulted in $r=0.910$ with $p$-value $=0.001$. Therefore, the covariance structure between the force components cannot be neglected.

Table 7 Confirmation runs

\begin{tabular}{cccc}
\hline Run order & $l_{\text {to }}(\mathrm{mm})$ & $F_{a}[\mathrm{~N}]$ & $F_{r}[\mathrm{~N}]$ \\
\hline 1 & 38 & 176.8 & 86.4 \\
2 & 46 & 163.6 & 84.9 \\
3 & 30 & 169.9 & 90.3 \\
4 & 38 & 169.0 & 85.9 \\
5 & 46 & 174.9 & 94.1 \\
6 & 46 & 176.2 & 93.9 \\
7 & 38 & 175.9 & 89.1 \\
8 & 30 & 174.4 & 92.2 \\
9 & 30 & 173.8 & 92.9 \\
\hline
\end{tabular}




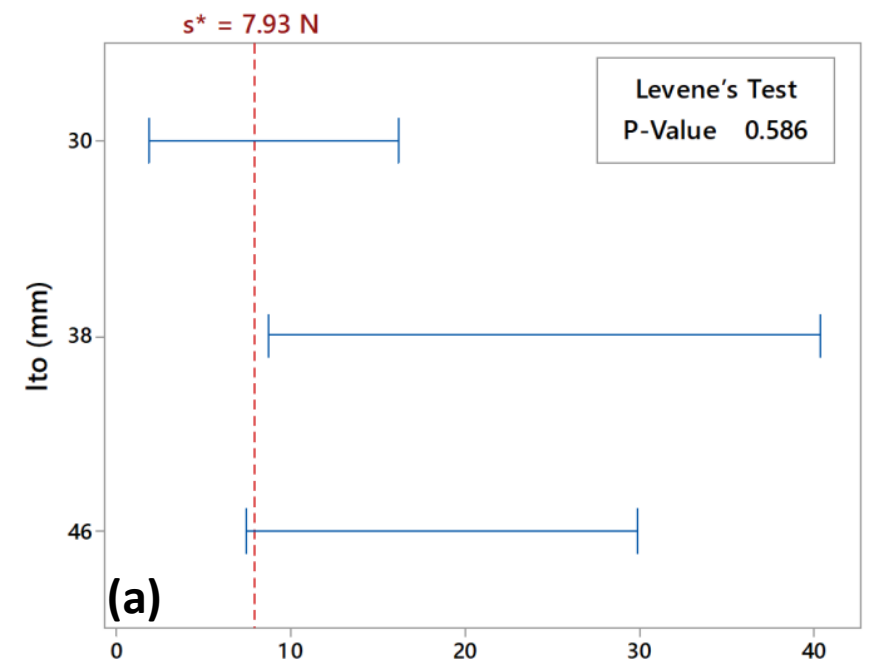

If intervals do not overlap, the corresponding stdevs are significantly different.

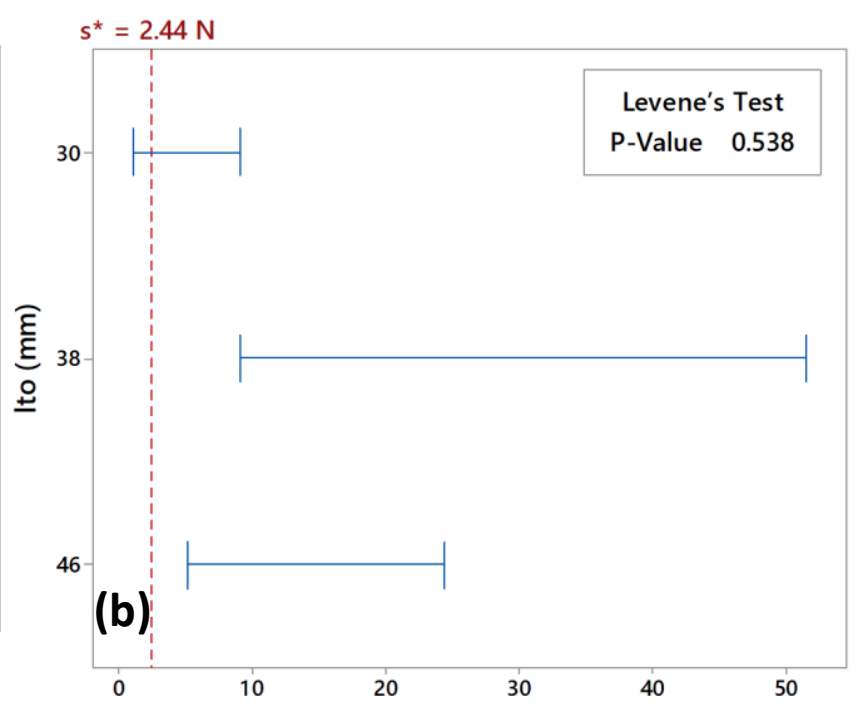

Fig. 12 Levene's test for equal variances. Multiple comparison intervals for the standard deviation, $\alpha=0.05$. (a) $F_{a}$ vs $l_{t o}$ and (b) $F_{r}$ vs $l_{t o}$

To assure homoscedasticity, i.e., the robustness of the Pareto optimal vector $\mathbf{x}_{w=0.5}^{*}=[-1.36 ; 0.57 ; 0.81]$ regarding the $l_{t o}$ variation, the Levene's test was performed for $F_{a}$ and $F_{r}$ considering the noise factor $l_{t o}$, and is resumed in Fig. 12 . As the null hypothesis of the Levene's test attests the variance equality and the $p$-values are higher than the significance level, $p$-value $>\alpha=0.05$, it cannot be rejected the homoscedasticity of the responses with consideration to the noise factor $l_{t o}$. In practical terms, it means that the variance regarding the tool overhang length variation is homogeneous in this robust Pareto optimal solution. However, the homogeneity of means regarding this noise factor should also be attested. Then, the MANOVA analysis was performed, considering the three tool overhang length levels $(30 ; 38 ; 46)$ and $F_{a}$ and $F_{r}$ as responses, to assure more power on conclusions, taking into consideration not only the variances but also the covariance between the responses. Table 8 presents the MANOVA analysis of the confirmation runs considering the correlated responses $F_{a}$ and $F_{r}$ against the noise factor $l_{t o}$. It can be stated that the noise factor effect is not significant on the correlated responses since the $p$-values for the three criteria are higher than the significance level, i.e., $p$-value $>\alpha=$ 0,05 . This means that, considering the optimal vector $\mathbf{x}_{w=0.5}^{*}=[-1.36 ; 0.57 ; 0.81]$, the process is robust to the tool overhang length variation.

Fig. 13 shows the confidence intervals for the confirmation runs for $F_{a}$ and $F_{r}$, considering the three different noise factor levels and the robust optimization results. In red reference lines, the predicted robust optimal mean levels are depicted, $F_{a}{ }^{*}=167.9 \mathrm{~N}$ and $F_{r}{ }^{*}=76.6 \mathrm{~N}$. For $F_{a}$, the predicted value was inside of the three confidence intervals with consideration to tool overhang length levels. For $F_{r}$, the predicted mean value was inside of only the confidence interval for $l_{t o}=38 \mathrm{~mm}$. However, the Pareto optimal value for $F_{r}{ }^{*}$ also presents a confidence interval associated with its standard deviation $S D_{z}\left[F_{r}\right]=2.4 \mathrm{~N}$. 

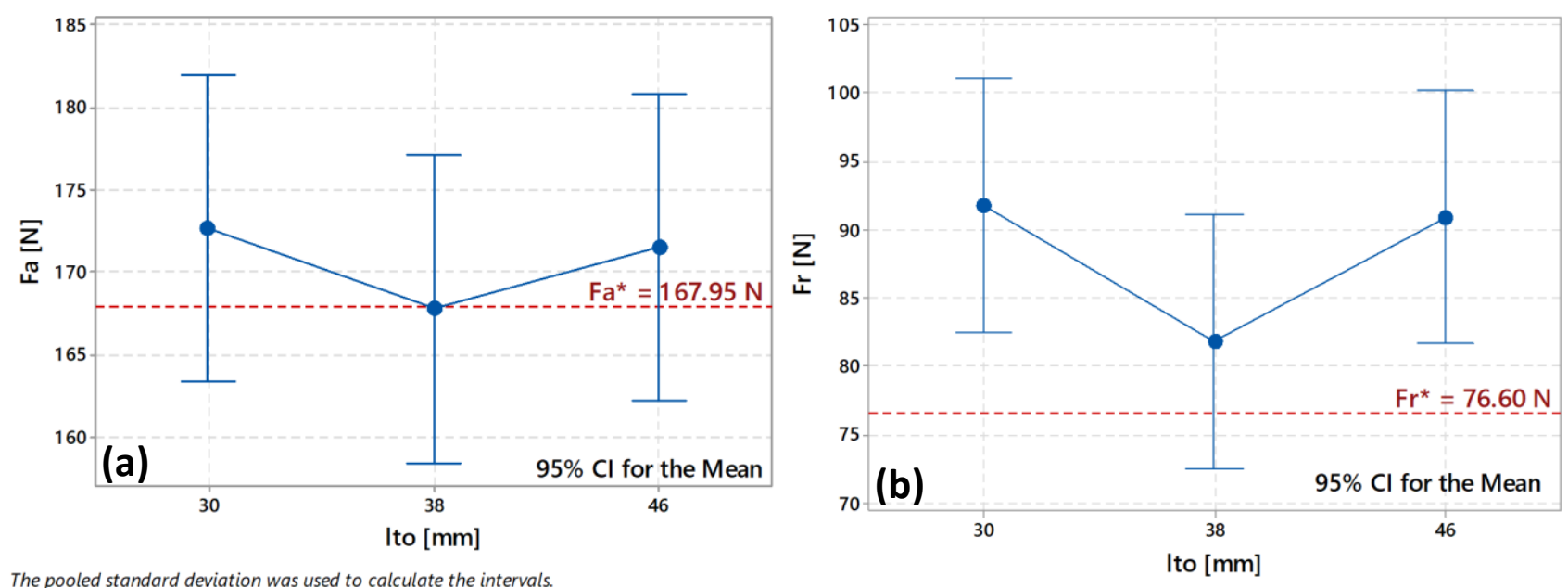

Fig. 13 Interval Plot of (a) $F_{a}$ vs $l_{t o}$ and (b) $F_{r}$ vs $l_{t o}$

Table 8 MANOVA for $F_{a}$ and $F_{r}$ vs $l_{t o}$

\begin{tabular}{llllll}
\hline \multirow{2}{*}{ Criterion } & \multirow{2}{*}{ Test Statistic } & $\mathrm{F}$ & \multicolumn{2}{l}{$\mathrm{DF}$} & \multirow{2}{*}{$\mathrm{P}$} \\
\cline { 4 - 6 } & & & Num & Denom & \\
\hline Wilks' & 0.27 & 2.30 & 4 & 10 & 0.131 \\
Lawley-Hotelling & 2.66 & 2.66 & 4 & 8 & 0.112 \\
Pillai's & 0.74 & 1.74 & 4 & 12 & 0.205 \\
Roy's & 2.65 & & & & \\
$\mathrm{~s}=2 \quad \mathrm{~m}=-0.5$ & $\mathrm{n}=1.5$ & & & & \\
\hline
\end{tabular}

The confirmation runs showed that the helical milling process of the aluminum alloy $\mathrm{Al} 7075$, considering the correlated responses $F_{a}$ and $F_{r}$, is robust to the tool overhang length variation considering the optimal vector $\mathbf{x}_{w=0.5}^{*}$. The other Pareto optimal points obtained through the $\mathrm{MMSE}_{\mathrm{W}}-\mathrm{NBI}$ method are also robust to the considered noise factor. Obviously, as the trade-off between mean and variance of the multivariate response WPC was explored by an equispaced Pareto frontier obtained through the $\mathrm{MMSE}_{\mathrm{W}} \mathrm{NBI}$ method, the equispaced Pareto points allow to the decision maker the possibility of choosing the desired balance between mean and variance of the correlated responses, which is very important for manufacturing engineers and for multivariate optimization researchers.

\subsection{Cutting force models in the workpiece coordinate system and time domain}

It is also important to set-up cutting force models in the workpiece coordinate system. In the present approach, the mean and variance models in the tool coordinate system are presented to assure robustness regarding the tool the overhang length variation. The models in the workpiece coordinate system are derived from the models in the tool coordinate system. Therefore, robustness is also achieved in the workpiece coordinate system.

As $F_{z}=F_{a}$, the models for mean and variance for $F_{z}$ are strictly equal to the Eq. (35) and Eq. (36), as presented in Eq. (39) and Eq. (40).

$$
\begin{aligned}
& E_{z}\left(F_{z}\right)=242.575+43.100 f_{z a}+7.049 f_{z t}+2.905 v_{c}-9.472 f_{z a}^{2}-7.663 f_{z t}^{2}-7.882 v_{c}^{2}-6.001 f_{z a} \times f_{z t} \\
& +2.000 f_{z a} \times v_{c}+0.393 f_{z t} \times v_{c} \\
& V_{z}\left(F_{z}\right)=27.642-47.511 f_{z a}-35.850 f_{z t}-20.050 v_{c}+21.271 f_{z a}^{2}+12.110 f_{z t}^{2}+3.788 v_{c}^{2}+32.100 f_{z a} \times f_{z t} \\
& +17.953 f_{z a} \times v_{c}+13.546 f_{z t} \times v_{c}
\end{aligned}
$$

The models for $F_{x}$ and $F_{y}$ are derived regarding $F_{r}$, Eq. (41). Since $F_{r}=\sqrt{F_{x}^{2}+F_{y}{ }^{2}}$, leaving the gap between $F_{x}$ and $F_{y}$ aside. 
$F_{x}=F_{y}=\frac{1}{\sqrt{2}} F_{r}$

Applying the mean operator, one obtains:

$E_{z}\left[F_{x}\right]=E_{z}\left[F_{y}\right]=E_{z}\left[\frac{1}{\sqrt{2}} F_{r}\right]=\frac{1}{\sqrt{2}} E_{z}\left[F_{r}\right]$

These models in time domain are defined as follows:

$E_{z}\left[F_{x}\right]=\frac{1}{\sqrt{2}} E_{z}\left[F_{r}\right] \cos \left(\omega_{o} \cdot t\right)$

$E_{z}\left[F_{y}\right]=\frac{1}{\sqrt{2}} E_{z}\left[F_{r}\right] \sin \left(\omega_{o} \cdot t\right)$

where $\omega_{o}$, in $[\mathrm{rad} / \mathrm{s}]$, is the angular velocity of the orbital feed motion, and $t$, in $[s]$, is the time. The angular velocity of the orbital feed motion $\omega_{o}$ is calculated according to the Eq. (45), where the orbital rotation speed $n_{o}$ is obtained according to Eq. (46) [13].

$\omega_{o}=\frac{2 \pi n_{o}}{60}$

$n_{o}=\frac{v_{f h t}}{\pi \cdot D_{h}}$

Finally, replacing Eq. (37) into Eq. (43) and Eq. (44), the models for mean of $F_{x}$ and $F_{y}$ are explicitly exposed in Eq. (47) and Eq. (48).

$E_{z}\left(F_{x}\right)=\frac{1}{\sqrt{2}}\left\{\begin{array}{l}194.422+72.028 f_{z a}-12.407 f_{z t}-1.429 v_{c}-2.059 f_{z a}^{2}-2.134 f_{z t}^{2}+1.205 v_{c}^{2}+7.009 f_{z a} \times f_{z t} \\ +0.973 f_{z a} \times v_{c}-3.479 f_{z t} \times v_{c}\end{array}\right\} \cos \left(\omega_{o} \cdot t\right)$
$E_{z}\left(F_{y}\right)=\frac{1}{\sqrt{2}}\left\{\begin{array}{l}194.422+72.028 f_{z a}-12.407 f_{z t}-1.429 v_{c}-2.059 f_{z a}^{2}-2.134 f_{z t}^{2}+1.205 v_{c}^{2}+7.009 f_{z a} \times f_{z t} \\ +0.973 f_{z a} \times v_{c}-3.479 f_{z t} \times v_{c}\end{array}\right\} \sin \left(\omega_{o} \cdot t\right)$

Analogously, the variance operator may be applied in Eq. (41). By doing so, it may be obtained:

$V_{z}\left[F_{x}\right]=V_{z}\left[F_{y}\right]=V_{z}\left[\frac{1}{\sqrt{2}} F_{r}\right]=\frac{1}{2} V_{z}\left[F_{r}\right]$

The variance for $F_{x}$ and $F_{y}$ may not be obtained in the time domain since variance cannot assume negative values. Explicitly, Eq. (50) provides the variance for $F_{x}$ and $F_{y}$. This equation provides an estimate of the error in the cutting force components on $x y$ plane regarding the tool overhang length variation.

$V_{z}\left(F_{x}\right)=V_{z}\left(F_{y}\right)=\frac{1}{2}\left\{\begin{array}{l}4.594+5.327 f_{z a}-11.521 f_{z t}-0.249 v_{c}+2.221 f_{z a}^{2}+10.388 f_{z t}^{2}+0.005 v_{c}^{2}-9.606 f_{z a} \times f_{z t} \\ -0.208 f_{z a} \times v_{c}+0.449 f_{z t} \times v_{c}\end{array}\right\}$

Considering the optimal vector $\mathbf{x}_{w=0.5}^{*}$, Fig. 14 shows the predicted cutting force in the workpiece coordinate system according to Eq. (39), Eq. (47) and Eq. (48) together with the experimental levels of one of the confirmation runs. As it may be observed, the predicted values were close to the experimental values. However, the propagation of error proposed in RPD with combined array represents in the equation a part of variability regarding the experimental error, which may take into account another noise factor not considered in the present study. Then, the proposed method may be applied in the helical milling considering others noise factors of this process to allow predicting the variability of cutting forces more precisely. It is usual to provide the percentage error of cutting force models regarding experimentally obtained values. However, in the present approach, there is a model to predict the error, i.e., the variance model for each 
cutting force component. As these models are in function of helical milling parameters, the error of the proposed approach will vary according to the selected Pareto optimal vector in the experimental region. It means that there are experimental levels, which were achieved with the proposed approach MMSE $\mathrm{W}_{\mathrm{W}} \mathrm{NBI}$, obtained to achieve low variance levels. For the example exposed in Fig. 14, the percentage errors in $F_{x}, F_{y}$ and $F_{z}$ were $1.05 \%, 0.15 \%$ and $0.67 \%$, respectively. In other experimental regions, where the process is not robust regarding the tool overhang length variation, these levels of error may not be guaranteed. Then, it is important to consider the variance models to achieve lower error levels of the mean models regarding experimental levels. Besides, these models work inside the experimental region, i.e., respecting the constraint $\mathbf{x}^{\mathbf{T}} \mathbf{x}=f_{z a}{ }^{2}+f_{z t}{ }^{2}+v_{c}{ }^{2} \leq \rho^{2}=2.828$, in coded units.

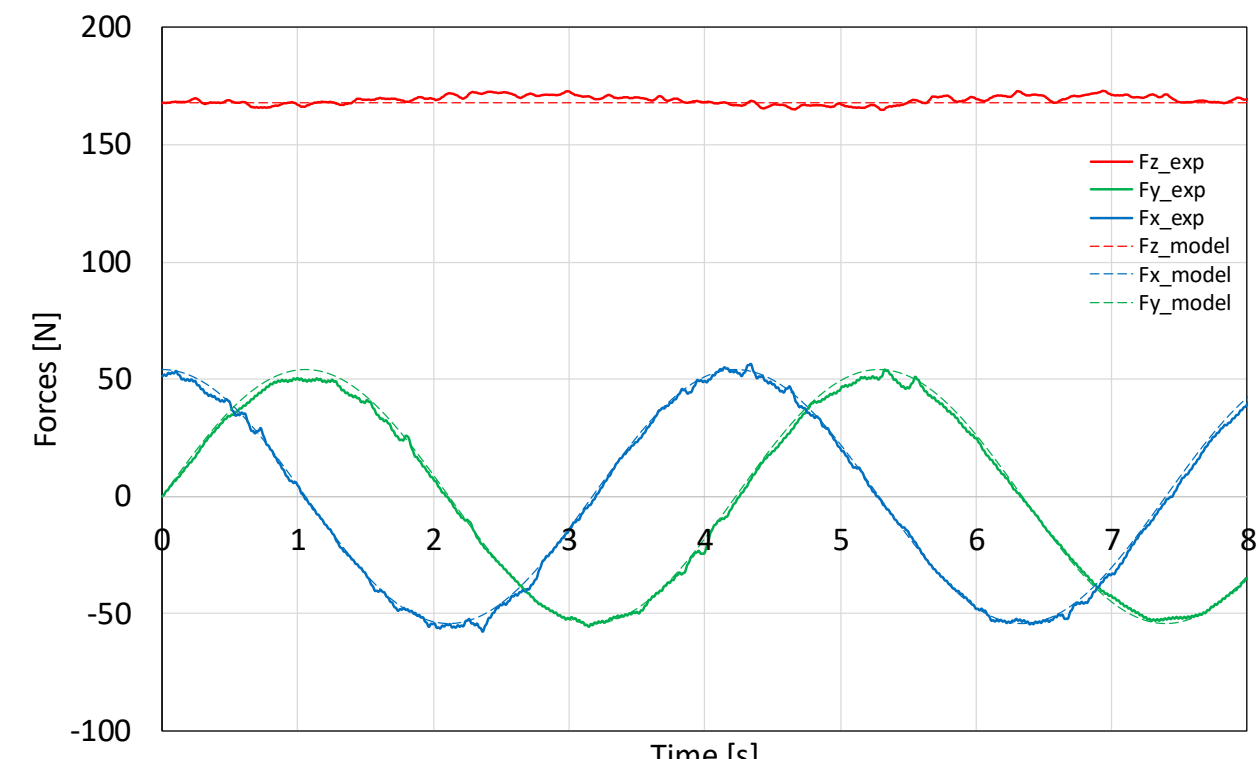

Fig. 14 Comparison of experimental and modelled cutting force in the workpiece coordinate system. $f_{z a}=2.75 \mu \mathrm{m} /$ tooth, $f_{z t}=79.3 \mu \mathrm{m} /$ tooth and $v_{c}=66.2 \mathrm{~m} / \mathrm{min}$

\section{Conclusions and future works}

The present work presented a new multivariate robust modelling and optimization method. The method guarantees the multivariate modelling and optimization of bias and variance of the axial and radial cutting force components of the helical milling process of the aluminum alloy Al 7075. Also, there is no loss of information, through the optimization of the mean and variance equations of the weighted principal component.

The tool overhang length was set as a noise factor since in cavity machining there are specific workpiece geometries that constrain this factor. To complete the set of independent variables, the axial and tangential feed per tooth and cutting velocity were chosen as controllable factors.

The MMSE ${ }_{W}-N B I$ 's combination was proposed to allow the replication of the method. The weighted principal component technique was carried out to avoid loss of information and to deal with the correlation problem between the axial and radial cutting force components.

The results showed good determination coefficients for the response surface models of the weighted principal component and the axial and radial cutting force components. The NBI method optimized the mean and variance equations of the weighted principal component. Optimal vectors were replaced in the mean and variance equations of the helical milling outcomes considered in this paper, leading to their robust optimal solutions. Pareto frontiers were plotted considering mean and standard deviation estimates of the weighted principal component, confirming that the MMSE $\mathrm{W}^{-}$ NBI method is efficient in generating equiespaced Pareto solutions.

Confirmation runs were carried out on the optimal robust vector $\mathbf{x}_{w=0.5}^{*}=[2.7 \mu \mathrm{m} /$ tooth; $79.3 \mu \mathrm{m} /$ tooth; 66.2 $\mathrm{m} / \mathrm{min}$ ] with three tool overhang length levels $(30 ; 38 ; 46)[\mathrm{mm}]$ and three replications. The Levene's test revealed that it cannot be rejected the homoscedasticity of the outcomes with consideration to the noise factor, i.e., the robustness of the Pareto optimal vector $\mathbf{x}_{w=0.5}^{*}$ was confirmed. To assure more power on conclusions, the multivariate analysis of variance (MANOVA) was performed considering the confirmation runs; the results showed that the noise factor effect is not significant on the correlated outcomes, considering the optimal vector.

Besides obtaining mean and variance cutting force models in the tool coordinate system, mean and variance models for the cutting force components were also obtained in the workpiece coordinate system in the time domain. Considering the optimal vector $\mathbf{x}_{w=0.5}^{*}$, the percentage errors in $F_{x}, F_{y}$ and $F_{z}$ were only $1.05,0.15$ and $0.67 \%$, respectively. The 
variance models of the cutting forces regarding the tool overhang length is an important achievement since the experimenter may apply the Pareto optimal experimental solutions to achieve lower error levels for mean models obtained for cutting force components.

For future works, other helical milling responses could be taken into consideration when performing the proposed multivariate robust and optimization method like roughness and geometrical error responses. Furthermore, extra helical milling noise factors could be explored like tool wear, workpiece hardness, workpiece grain sizes, and others. Different multivariate methods could be explored for multivariate statistical analysis. Finally, additional optimization methods could be tested in comparison with NBI and MSE. Methods like normalized normal constraint (NNC), $\varepsilon$-constraint, and others that allow the inclusion of multivariate techniques in their routines.

Acknowledgements The authors gratefully acknowledge CNPq, CAPES, FAPEMIG and DEMEC/UFSJ for supporting this research. The first author acknowledges CAPES for the PDSE grant, process $\mathrm{n}^{\mathrm{o}}$. 88881.133263/2016-01.

\section{References}

1. Iyer R, Koshy P, Ng E (2007) Helical milling: an enabling technology for hard machining precision holes in AISI D2 tool steel. Int J Mach Tools Manuf 47:205-210.

2. Pilný L, De Chiffre L, Píška M, Villumsen MF (2012) Hole quality and burr reduction in drilling aluminum sheets. CIRP J Manuf Sci Technol 5:102-107.

3. Sheth S, George PM (2016) Experimental investigation, prediction and optimization of cylindricity and perpendicularity during drilling of WCB material using grey relational analysis. Precis Eng 45:33-43.

4. Brinksmeier E, Fangmann S, Rentsch R (2011) Drilling of composites and resulting surface integrity. CIRP Ann Manuf Technol 60:57-60.

5. Li H, He G, Qin X, Wang G (2014) Tool wear and hole quality investigation in dry helical milling of Ti-6Al-4V alloy. Int J Adv Manuf Technol 71:1511-1523.

6. Li Z, Liu Q (2012) Surface topography and roughness in hole-making by helical milling. Int J Adv Manuf Technol 66:1415-1425.

7. Qin X, Gui L, Li H, Rong B, Wang D, Zhang H, et al (2012) Feasibility study on the minimum quantity lubrication in high-speed helical milling of Ti-6Al-4V. J Adv Mech Des Syst Manuf 6:1222-1233.

8. Brinksmeier E, Fangmann S, Meyer I (2008) Orbital drilling kinematics. Prod Eng Res Devel 2:277-283.

9. Sasahara H, Kawasaki M, Tsutsumi M (2008) Helical feed milling with MQL for boring of aluminum alloy. J Adv Mech Des Syst Manuf 2:1030-1040.

10. Fang Q, Pan Z, Fei S, Xie X, Ke Y (2015) A novel helical milling end-effector and its application. IEEE/ASME Trans Mechatron 20:3112-3122.

11. Tanaka H, Ohta K, Takizawa R, Yanagi K (2012) Experimental study on tilted planetary motion drilling for CFRP. Procedia CIRP 1:443-448.

12. Denkena B, Boehnke D, Dege JH (2008) Helical milling of CFRP-titanium layer compounds. CIRP J Manuf Sci Technol 1:64-69.

13. Pereira RBD, Brandão LC, Paiva AP, Ferreira JR, Davim JP (2017) A review of helical milling process. Int J Mach Tools Manuf 120:27-48.

14. Ventura CEH, Hassui A (2013) Modeling of cutting forces in helical milling by analysis of tool contact angle and respective depths of cut. Int J Adv Manuf Technol 68:2311-2319.

15. Wang H, Qin X, Ren C, Wang Q (2011) Prediction of cutting forces in helical milling process. Int J Adv Manuf Technol 58:849-859.

16. Corne R, Nath C, El Mansori M, Kurfess T (2017) Study of spindle power data with neural network for predicting real-time tool wear/breakage during inconel drilling. J Manuf Syst 43:287-295.

17. Haiyan W, Xuda Q (2016) A mechanistic model for cutting force in helical milling of carbon fiber-reinforced polymers. Int J Adv Manuf Technol 82:1485-1494.

18. Tian Y, Liu Y, Wang F, Jing X, Zhang D, Liu X (2017) Modeling and analyses of helical milling process. Int J Adv Manuf Technol 90:1003-1022.

19. Wang H, Qin X, Li H, Tan Y (2016) A comparative study on helical milling of CFRP/Ti stacks and its individual layers. Int J Adv Manuf Technol 5:1973-1983.

20. Shan Y, He N, Li L, Zhao W, Qin X (2011) Orbital milling hole of aerospace Al-Alloy with big pitch. Trans of Tianjin Univ 17:329-335.

21. Liu C, Wang G, Dargusch MS (2012) Modelling, simulation and experimental investigation of cutting forces during helical milling operations. Int J Adv Manuf Technol 63:839-850.

22. Rey PA, LeDref J, Senatore J, Landon Y (2016) Modelling of cutting forces in orbital drilling of titanium alloy Ti6Al-4V. Int J Mach Tools Manuf 106:75-88. 
23. Li ZL, Ding Y, Zhu LM (2017) Accurate cutting force prediction of helical milling operations considering the cutter runout effect. Int J Adv Manuf Technol 1-12.

24. Mishra V, Khan GS, Chattopadhyay KD, Nand K, Sarepaka RV (2014) Effects of tool overhang on selection of machining parameters and surface finish during diamond turning. Measurement 55:353-361.

25. Kiyak M, Kaner B, Sahin I, Aldemir B, Cakir O (2010) The dependence of tool overhang on surface quality and tool wear in the turning process. Int J Adv Manuf Technol 51:431-438.

26. Rama Kotaiah K, Srinivas J, Sekar M (2009) Prediction of optimal stability states in inward-turning operation using neurogenetic algorithms. Int J Adv Manuf Technol 45:679-689.

27. Kull Neto H, Diniz AE, Pederiva R (2016) Influence of tooth passing frequency, feed direction, and tool overhang on the surface roughness of curved surfaces of hardened steel. Int J Adv Manuf Technol 82:753-764.

28. Basu S, Dan PK, Thakur A (2014) Experimental design in soap manufacturing for optimization of fuzzified process capability index. J Manuf Syst 33:323-334.

29. Myers RH, Khuri AI, Vining G (1992) Response surface alternatives to the Taguchi robust parameter design approach. Am Stat 46:131-139.

30. Nair VN, Abraham B, Mackay J, Box G, Kacker RN, Lorenzen TJ, et al. (1992) Taguchi's parameter design: a panel discussion. Technometrics 34:127-161.

31. Welch W, Yu T, Kang SM, Sacks J (1990) Computer experiments for quality control by parameter design. J Qual Technol 22:15-22.

32. Shoemaker AC, Tsui KL, Wu CFJ (1991) Economical experimentation methods for robust design. Technometrics 33:415-427.

33. Box G, Jones S (1992) Designing products that are robust to the environment. Total Qual Manag 3:265-282.

34. Box G (1998) Signal-to-noise ratios, performance criteria, and transformations. Technometrics 30:1-17.

35. Lin DKJ, Tu W (1995) Dual response surface optimization. J Qual Technol 27:34-39.

36. Köksoy O (2006) Multiresponse robust design: mean square error (MSE) criterion. Appl Math Comput 175:17161729.

37. Paiva AP, Paiva EJ, Ferreira JR, Balestrassi PP, Costa SC (2009) A multivariate mean square error optimization of AISI 52100 hardened steel turning. Int J Adv Manuf Technol 43:631-643.

38. Paiva AP, Campos PH, Ferreira JR, Lopes LGD, Paiva EJ, Balestrassi PP (2012) A multivariate robust parameter design approach for optimization of AISI 52100 hardened steel turning with wiper mixed ceramic tool. Int J Refract Met Hard Mater 30:152-163.

39. Paiva AP, Gomes JHF, Peruchi RS, Leme RC, Balestrassi PP (2014) A multivariate robust parameter optimization approach based on principal component analysis with combined arrays. Comput Ind Eng 74:186-198.

40. Box GEP, Hunter WG, Macgregor JF, Erjavec J (1973) Some problems associated with the analysis of multiresponse data. Technometrics 15:33-51.

41. Johnson RA, Wichern DW (2007) Applied multivariate statistical analysis. 6th ed. Pearson Prentice-Hall, New Jersey.

42. Liao HC (2006) Multi-response optimization using weighted principal component. Int J Adv Manuf Technol 27:720-725.

43. Rencher AC (2002) Methods of multivariate analysis. 2nd ed. John Wiley \& Sons, New York.

44. Wu FC, Chyu CC (2004) Optimization of correlated multiple quality characteristics robust design using principal component analysis. J Manuf Syst 23:134-143.

45. Das I, Dennis JE (1998) Normal-boundary intersection: a new method for generating the pareto surface in nonlinear multicriteria optimization problems. SIAM J Optim 8:631-657.

46. Ganesan T, Vasant P, Elamvazuthi I (2013) Normal-boundary intersection based parametric multi-objective optimization of green sand mould system. J Manuf Syst 32:197-205.

47. Zhang Q, Liou JJ, McMacken J, Thomson J, Layman P (2001) Development of robust interconnect model based on design of experiments and multiobjective optimization. IEEE Trans Electron Devices 48:1885-1891.

48. Brito TG, Paiva AP, Ferreira JR, Gomes JHF, Balestrassi PP (2014) A normal boundary intersection approach to multiresponse robust optimization of the surface roughness in end milling process with combined arrays. Precis Eng 38:628-638.

49. Costa DMD, Paula TI, Silva PAP, Paiva AP (2016) Normal boundary intersection method based on principal components and Taguchi's signal-to-noise ratio applied to the multiobjective optimization of 12L14 free machining steel turning process. Int J Adv Manuf Technol 87:825-834.

50. Das I, Dennis JE (1996) Normal-boundary intersection: an alternate method for generating pareto optimal points in multicriteria optimization problems. NASA Contractor Report 201616.

51. Paiva AP, Costa SC, Paiva EJ, Balestrassi PP, Ferreira JR (2010) Multi-objective optimization of pulsed gas metal arc welding process based on weighted principal component scores. Int J Adv Manuf Technol 50:113-125.

52. Jia Z, Ierapetritou MG (2007) Generate Pareto optimal solutions of scheduling problems using normal boundary intersection technique. Comput Chem Eng 31:268-280. 
53. Lopes LGD, Brito, TG, Paiva AP, Peruchi RS, Balestrassi PP (2016) Robust parameter optimization based on multivariate normal boundary intersection. Comput Ind Eng 93:55-66.

54. Haggag AA (1981) A variant of the generalized reduced gradient algorithm for non-linear programming and its applications. Eur J Oper Res7:161-168.

55. Pereira RBD, Leite RR, Alvim AC, Paiva AP, Ferreira JR, Davim JP (2017) Multi-objective robust optimization of the sustainable helical milling process of the aluminum alloy Al 7075 using the augmented- enhanced normalized normal constraint method. J Clean Prod 152:474-496.

56. Li Z, Liu Q, Ming X, Wang X, Dong Y (2014) Cutting force prediction and analytical solution of regenerative chatter stability for helical milling operation. Int J Adv Manuf Technol 73:433-442. 